Research paper

\title{
Investigation into the apparent permeability and gas-bearing property in typical organic pores in shale rocks
}

\author{
Lili $\mathrm{Ji}^{\mathrm{a}}$, Mian Lin ${ }^{\mathrm{a}, \mathrm{b}, *}$, Wenbin Jiang ${ }^{\mathrm{a}, \mathrm{b}}$, Gaohui Cao ${ }^{\mathrm{a}, \mathrm{b}}$, Ji Zhou ${ }^{\mathrm{a}, \mathrm{b}}$, Chao Luo ${ }^{\mathrm{c}}$ \\ ${ }^{a}$ Key Laboratory for Mechanics in Fluid Solid Coupling Systems, Institute of Mechanics, Chinese Academy of Sciences, Beijing, 100190, China \\ ${ }^{\mathrm{b}}$ School of Engineering Science, University of Chinese Academy of Sciences, Beijing, 100190, China \\ ${ }^{\mathrm{c}}$ PetroChina, Southwest Branch, Chengdu, 61050, China
}

\section{A R T I C L E I N F O}

\section{Keywords:}

Typical organic pores

Apparent permeability in $\mathrm{OM}$

Gas-bearing property of $\mathrm{OM}$

High pressure and temperature

Rock compressibility

\begin{abstract}
A B S T R A C T
Although substantial advances have been made in investigating the storage and transport properties of shale gas in past decades, understanding of the flow characteristics and gas-bearing property in organic pores remains limited. In this paper, organic pores in the organic matter (OM) of the marine Longmaxi Formation are classified into six types based on the morphology and sizes of nanoscale pores. The structures of the six types of typical organic pores under high temperature and pressure are investigated based on the digital core compressibility method introduced in this paper. The results show that the pore-size distribution and connectivity of organic pores under high temperature and pressure are considerably different from those under conventional conditions. The apparent permeability of OM with different proportions of macropores, mesopores, and micropores are analyzed. The results demonstrate that the permeability of OM with Type 1 organic pores has the largest changes after compaction, whereas OM with Type 5 organic pores has a relatively smaller change. The regularities of the absolute adsorption amount and the free gas amount of OM with typical organic pores are also investigated. The absolute adsorption amount of OM decreases with the proportion of macropores, whereas the amount of free gas of OM increases. Finally, a chart of the absolute adsorption amount and the free gas amount of OM at different depths is given, which is helpful for estimating the gas-bearing capacity of OM and preliminary understanding the quality of a shale reservoir.
\end{abstract}

\section{Introduction}

Because of the world's ever-growing demand for energy, organicrich shale has become increasingly important. Differing from conventional reservoirs, shale rock has abundant tiny pores (at nanoscale), which are the main reason for the extremely low porosity and permeability of these rocks (Javadpour et al., 2007; Loucks et al., 2009; Reed and Ruppel, 2012; Yang et al., 2014; Akkutlu et al., 2015). Nanoscale pores, which are mainly in organic matter (OM), can significantly affect the storage and percolation of shales (Chen et al., 2016; Darabi et al., 2012; Guo et al., 2015; Javadpour, 2009; Sheng et al., 2016). Especially, shales of the Paleozoic Erathem, which are widely distributed in southern China, are deeply buried and have reached high maturity (Liu et al., 2015). The properties of organic pores in environments with high pressure and temperature are usually quite different from those with normal pressure and temperature. Thus, it is important to study the properties of typical organic pores in deep formations with high pressure and temperature.

Natural gas in shale is mainly stored in shale reservoirs as adsorbed gas on the surfaces of pores and free gas in pores, as well as a small amount of dissolved gas (Curtis, 2002). According to this pore classification of IUPAC, pores in shale have commonly been divided into three groups: macropores $(>50 \mathrm{~nm})$, mesopores $(2-50 \mathrm{~nm})$ and micropores $(<2 \mathrm{~nm}$ ) (Rouquerol et al., 1994). Xiong et al. (2017) have revealed that the free gas amount has a good correlation with the volume of macropores and mesopores. This is because that these two kinds of pores have large volume. Whereas at the same temperature and pressure, the density of the adsorbed gas in mesopores and micropores is much higher than that of the free gas, and this is because that mesopores and micropores account for most specific surface area. Ross and Bustin (2009) have shown that pore structure is one of the major factors that controls gas capacity. Organic pores in $\mathrm{OM}$ are composed of macropores, mesopores, and micropores. Different OM may have quite different proportions of three kinds of pores. Thus, the gas-bearing

\footnotetext{
* Corresponding author. Key Laboratory for Mechanics in Fluid Solid Coupling Systems, Institute of Mechanics, Chinese Academy of Sciences, Beijing, 100190, China.

E-mail address: linmian@imech.ac.cn (M. Lin).
} 


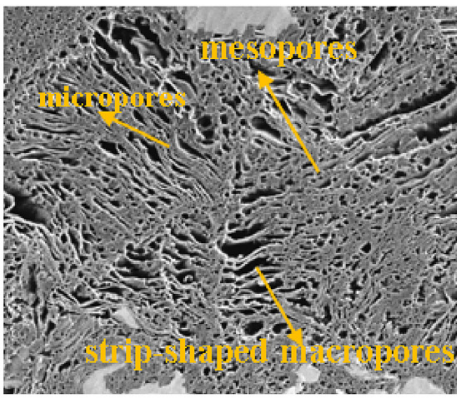

(a) Type 1

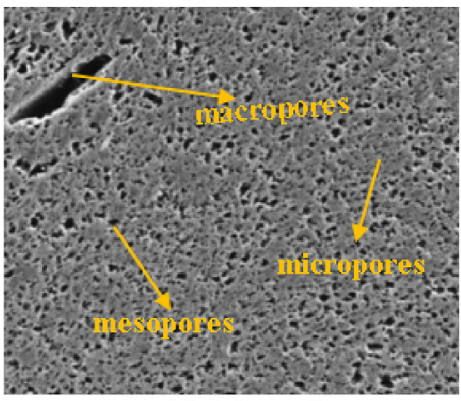

(d) Type 3

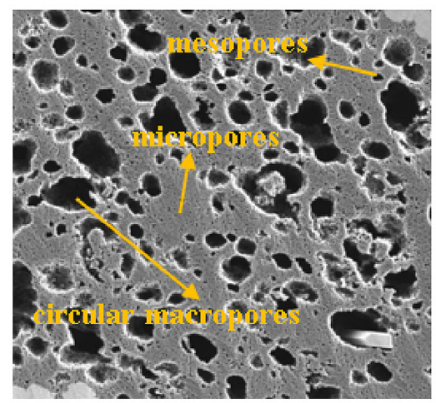

(b) Type 2

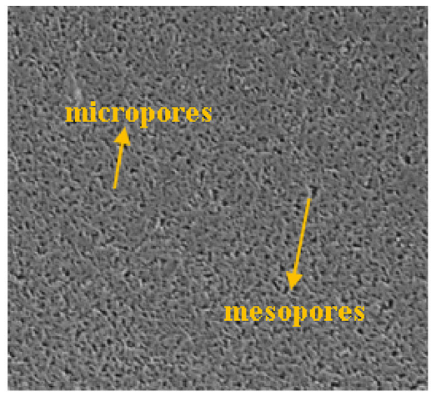

(e) Type 5

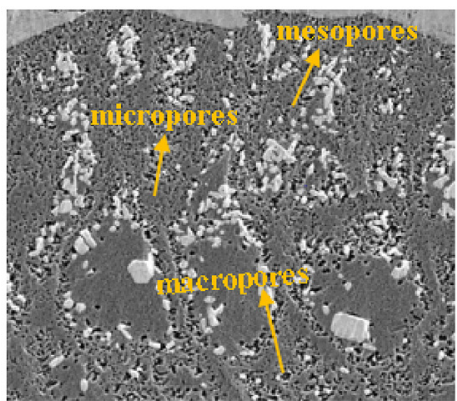

(c) Type 3

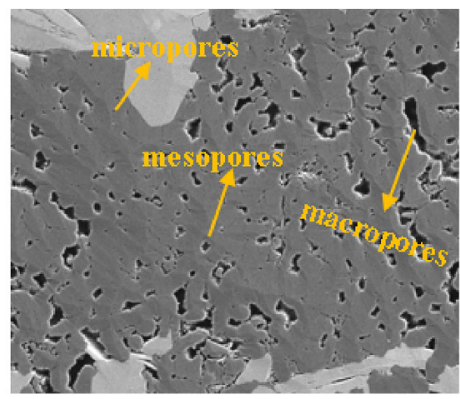

(f) Type 6

Fig. 1. SEM images of typical organic pores.

Table 1

The proportions of different pores for the six typical organic pores.

\begin{tabular}{lllllll}
\hline & Type 1 & Type 2 & Type 3 & Type 4 & Type 5 & Type 6 \\
\hline macropores & $40.25 \%$ & $67.11 \%$ & $34.45 \%$ & $7.30 \%$ & 0 & $15.82 \%$ \\
mesopores & $52.71 \%$ & $25.87 \%$ & $64.45 \%$ & $88.10 \%$ & $23.38 \%$ & $64.08 \%$ \\
micropores & $7.04 \%$ & $7.02 \%$ & $1.10 \%$ & $4.60 \%$ & $76.62 \%$ & $20.10 \%$ \\
\hline
\end{tabular}

property of different OM may differ considerably. It is necessary to study the gas-bearing property of $\mathrm{OM}$ with typical organic pores in shale reservoirs.

The transport of gas in shale is quite complex compared with conventional reservoirs as shale is rich in nanopores, which are primarily present in OM (Chen, 2016; Naraghi and Javadpour, 2015; Wang et al., 2016). Fathi and Akkutlu (2011) simulated the gas dynamics in organic matter based on the lattice Boltzmann method (LBM) and found that Darcy's law is only applicable for large organic pores ( $>100 \mathrm{~nm})$. Then, Akkutlu and Fathi (2012) separated kerogens from inorganic matrix by a newly proposed dual-porosity model. The result revealed that gas transport in kerogen was dominated by non-Darcy flow. By studying the effects of the apparent permeability at different pore pressures, Song et al. (2016) found that apparent permeability decreases with pore pressure when organic pore radii are smaller than $20 \mathrm{~nm}$ and remains nearly constant for organic pores larger than $20 \mathrm{~nm}$. The above research demonstrates that organic pores in OM play an important role in the transport of shale gas, and that the structure and size of organic pores can greatly affect the flow properties of gas. The structure and morphology of organic pores may change from OM to OM, and the gas flow in different OM may be quite different. Thus, it is necessary to investigate the regularities of the gas transport properties in typical organic pores.

As mentioned above, shales in southern China are usually deeply buried with reservoir conditions of high pressure and temperature. Dong et al. (2010) compared the stress sensitivity of shale matrix with sandstone based on experiments. They found that the apparent permeability of shale has a stronger stress dependence than that of sandstone. In 2015, a conceptual flow model, coupling molecular transport and stress dependence, was developed by Wasaki and Akkutlu (2015).
The sensitivity analysis in this paper revealed that as the effective stress increases, slit-shaped pores are closed, and the apparent permeability is significantly influenced by the stress. Later, to study the effect of effective stress on shales, Wu et al. (2016) proposed a gas transport model for organic-rich shales. The simulation results revealed that the influence of effective stress on gas transport is dependent on shale mechanical properties and gas transport mechanisms. However, in this model, two fitting coefficients of the permeability and porosity should be determined experimentally, and it is time-consuming to conduct experiments to measure these coefficients. Moreover, it is also not suitable for shales to use a constant stress-sensitive parameter to characterize the compression effect. Recently, Sheng et al. (2018) have introduced a compression coefficient to study the stress dependence of the apparent permeability of organic matter and inorganic matter. They found that for relatively large pores ( $>10 \mathrm{~nm}$ ), the effective stress had similar influence on the permeability of $\mathrm{OM}$ and inorganic matter. However, that paper only examined the effect of compressibility for individual pores with different sizes, and real shale has a multiscale pore structure that represents a network. Moreover, none of the above models considers the coupling of pore pressure and the effective stress during compaction. Overall, research on the compressibility of shales is still not a mature research field, and it is necessary to improve current methods to investigate the gas transport property and storage mechanisms of typical organic pores under high temperature and pressure.

The rest of our paper is organized as follows. First, we describe the characteristics of typical organic pores in $\mathrm{OM}$ and the digital core compressibility models. Then, the structure of six types of organic pores under high temperature and pressure are examined. The permeability and the ratio of the apparent permeability (kapp) and Darcy permeability (kd) are calculated. Then, the regularity of flow characteristics depending on different compositions of macropores, mesopores, and micropores are summarized. The gas-bearing property of OM with typical organic pores under high pressure and temperature is also investigated. The final section summarizes the paper and presents some important conclusions. 


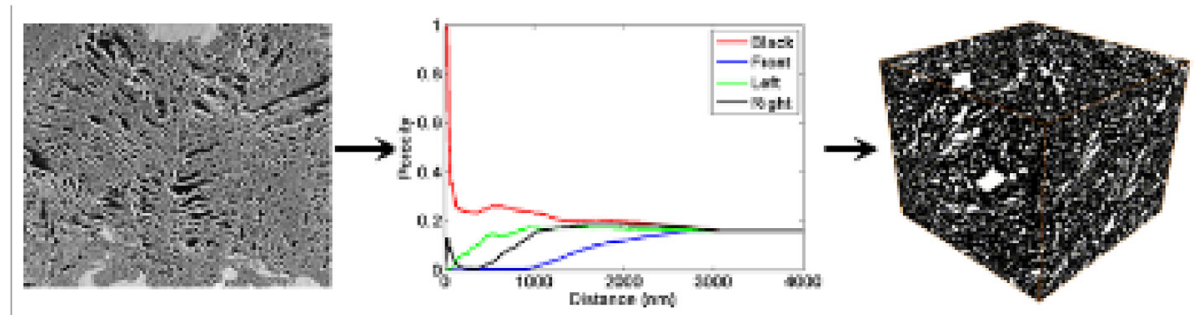

(a) Type 1

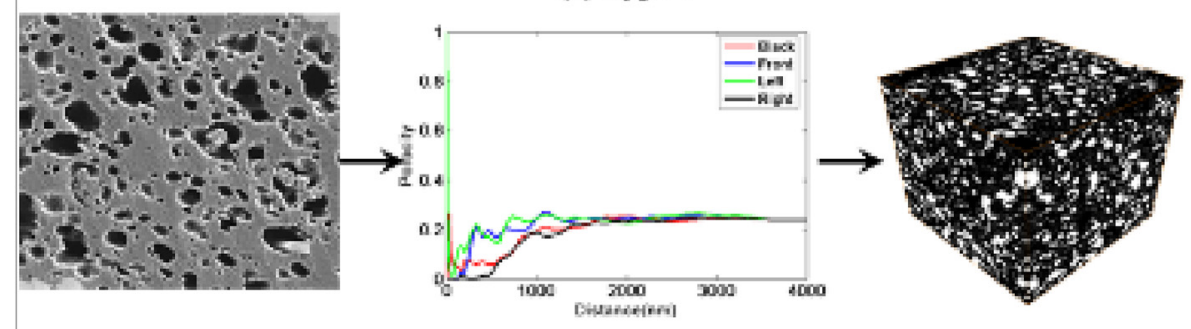

(b) Type 2

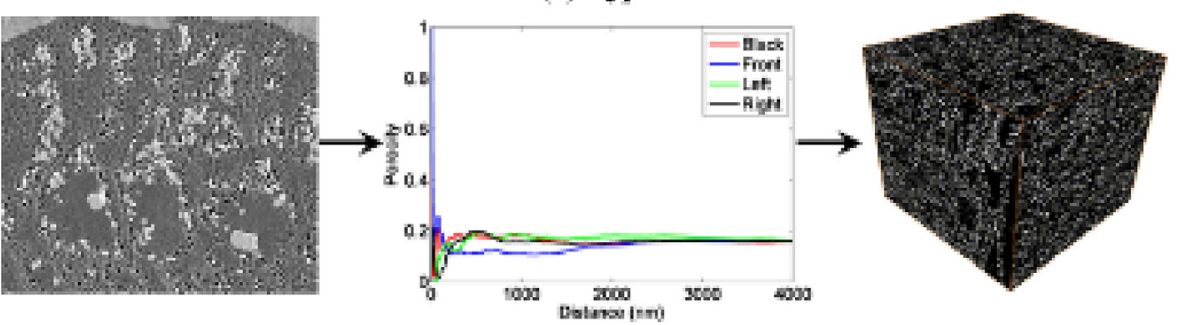

(c) Iype 3

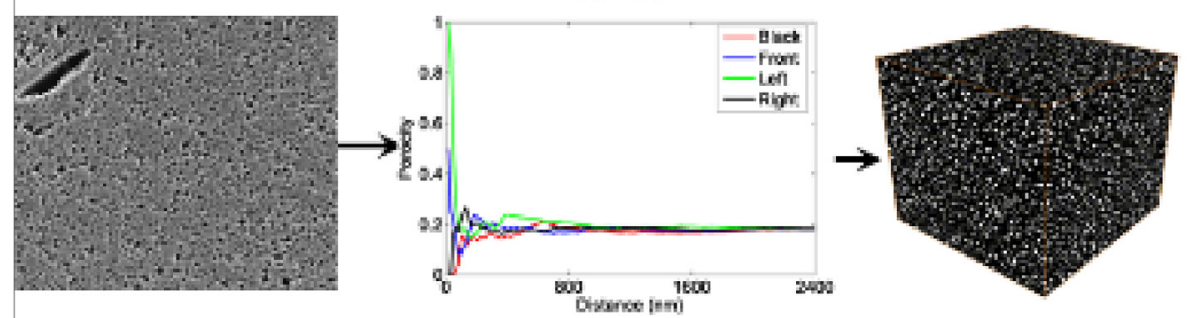

(d) Trpe 4

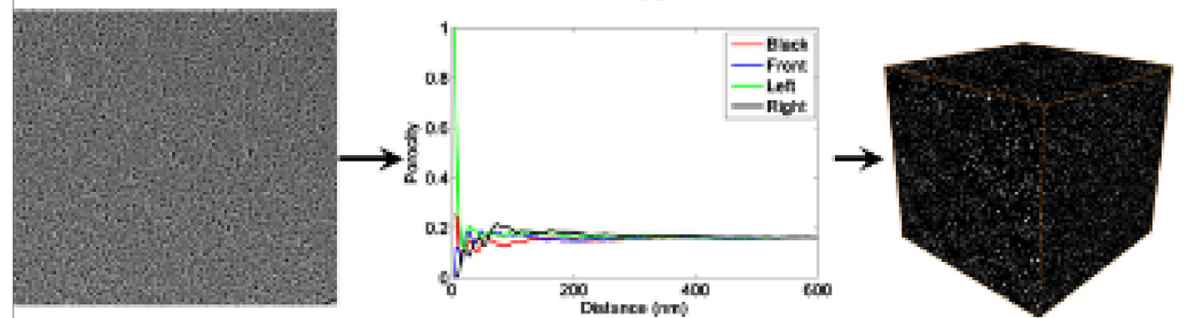

(e) Type 5

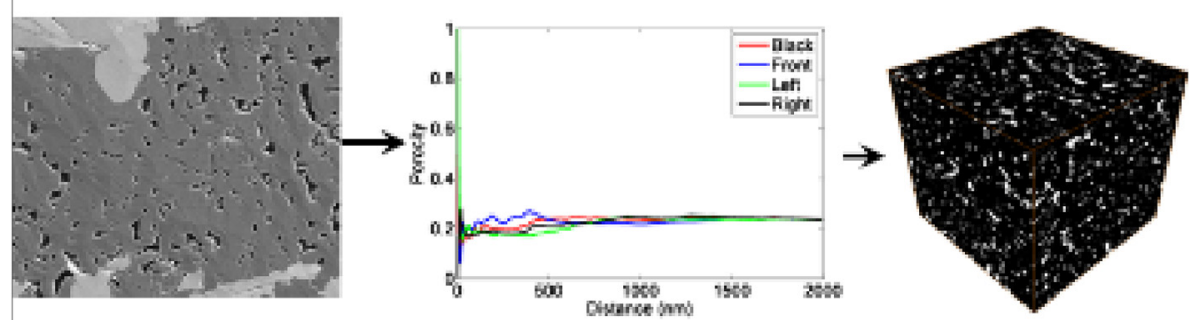

(f) Tvue 6

Fig. 2. The reconstructed digital cores of six types of the organic pores. The digital core is reconstructed based on the CCSIM-TSS method and the corresponding 2D images in Fig. 1. The sizes of the digital cores for Type 1-6 organic pores is $3.2 * 3.2 * 3.2 \mu \mathrm{m}^{3}, 3.2 * 3.2 * 3.2 \mu \mathrm{m}^{3}, 3.2 * 3.2 * 3.2 \mu \mathrm{m}^{3}$, $2.4 * 2.4 * 2.4 \mu \mathrm{m}^{3}, 0.6 * 0.6 * 0.6 \mu \mathrm{m}^{3}$ and $2.0 * 2.0 * 2.0 \mu \mathrm{m}^{3}$. 


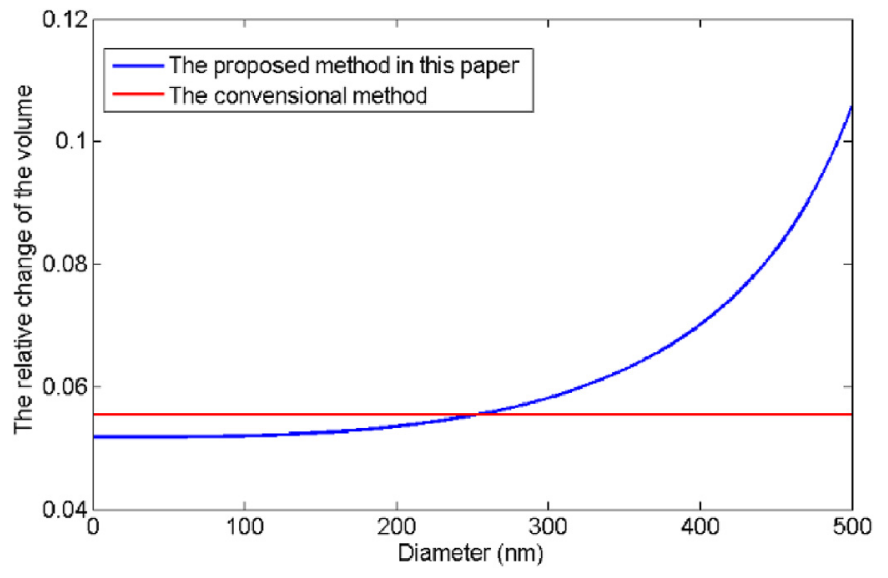

Fig. 3. Compacttion of pores with different sizes.

\section{The structure of typical organic pores}

\subsection{Typical organic pores}

For this paper, we chose typical organic pores that are widespread in OM of Lower Silurian Longmaxi Formation marine shale in Changning-Weiyuan region. Lower Silurian Longmaxi Formation was deposited in the shelf and intra-shelf low foreland setting with a thickness of 50-600 m. The lithology of the Lower Silurian Longmaxi Formation in Changning-Weiyuan region in the Sichuan Basin, China is mainly the marine organic-rich and quartz-rich siliceous shale. Moreover, the lithology on the upper of the formation is different from that in the lower section. The lower section, as the shale gas target, is

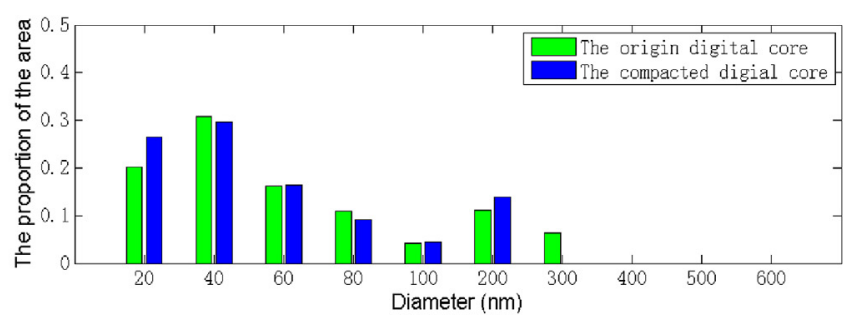

Type 1

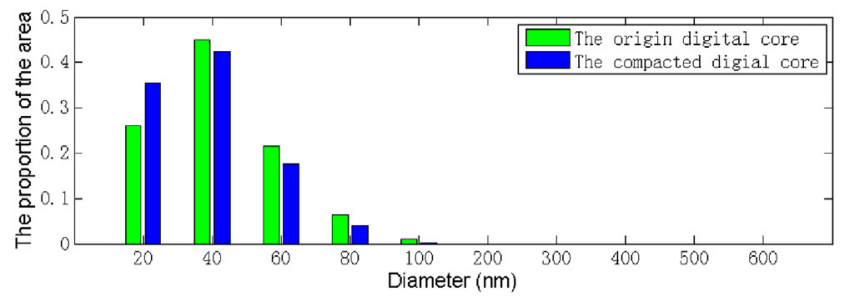

Type 3

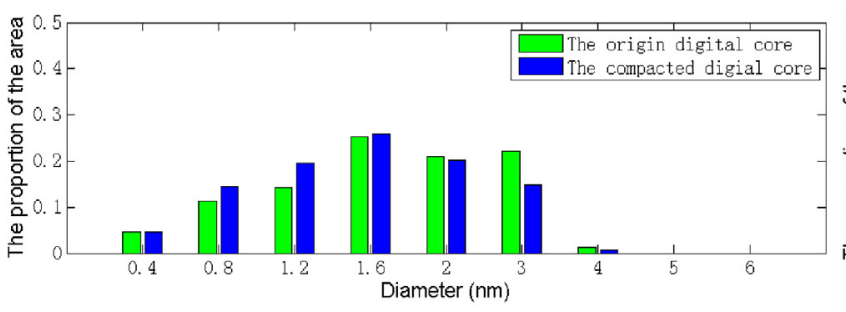

Type 5 characterized by black and grayish-black graptolite rich shale. And the upper section of the formation is composed of dark gray silty shale unequal interbedded with dark gray argillaceous limestone and siltstone laminas. After observing the SEM (Scanning Electron Microscope) and HIM (Helium-Ion-Microscope) images of many shale samples in this area, we found that the typical organic pores can be classified into six types according to the morphology of the nanoscale pores. Type 1 organic pores have large numbers of typical flocculated clay pores and some circular pores. The typical flocculated clay pores are strip-shaped macropores, and the circular pores are round mesopores and micropores. Type 2 organic pores have many round macropores and some mesopores and micropores. However, in Type 3 organic pores, the proportions of macropores and mesopores are almost equal, and the macropores and mesopores have a balanced development. In contrast with the above three types, there are a large portion of mesopores and a small portion of micropores in Type 4 organic pores, and Type 5 organic pores have a large portion of micropores and a small portion of mesopores. Type 6 organic pores have more mesopores and micropores than macropores. To investigate the structure, the apparent permeability and the gas-bearing property of typical organic pores, we design the images of typical organic pores by combination of the SEM and HIM images, as shown in Fig. 1. It should be pointed out that the resolutions of the images in Fig. 1 are all $0.5 \mathrm{~nm}$, and the sizes of Fig. $1 \mathrm{a}-\mathrm{f}$ are $4.0 * 4.0 \mu \mathrm{m}^{2}, 4.0 * 4.0 \mu \mathrm{m}^{2}, 4.0 * 4.0 \mu \mathrm{m}^{2}, 2.4 * 2.4 \mu \mathrm{m}^{2}, 0.6 * 0.6 \mu \mathrm{m}^{2}$ and $2.0 * 2.0 \mu \mathrm{m}^{2}$. Table 1 lists the proportions of the different pores for these six typical organic pore types. Generally, Type 1 organic pores have more strip-shaped macropores (40.25\%), and Type 2 organic pores have more round macropores (67.11\%). Type 3 organic pores have a balanced development of macropores and mesopores, and their proportions are almost equal. Type 4 organic pores have a large portion of mesopores (76.62\%). Type 5 organic pores have only mesopores and micropores, and Type 6 organic pores have more mesopores (64.08\%).

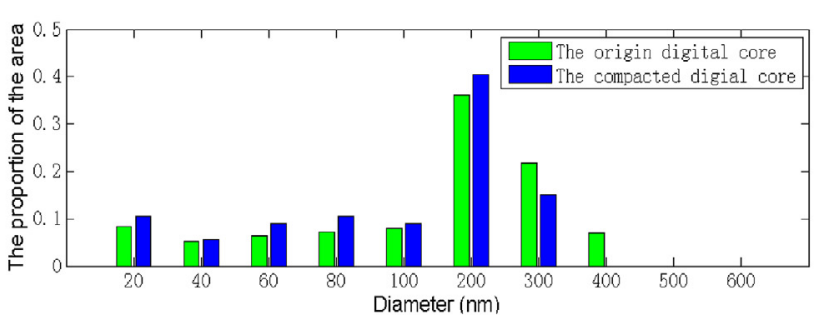

Type 2

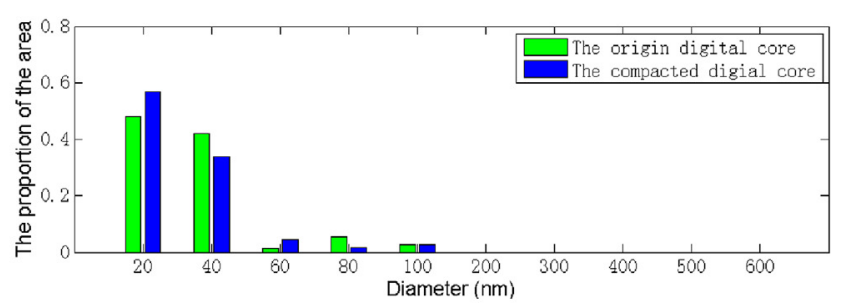

Type 4

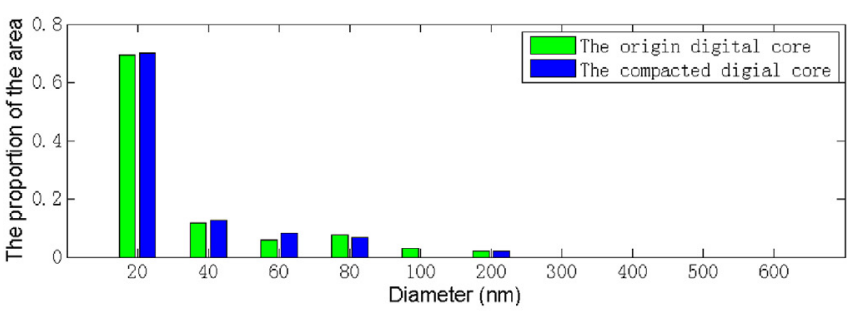

Type 6

Fig. 4. The pore-size distribution of each kind of typical organic pores before and after compaction. 


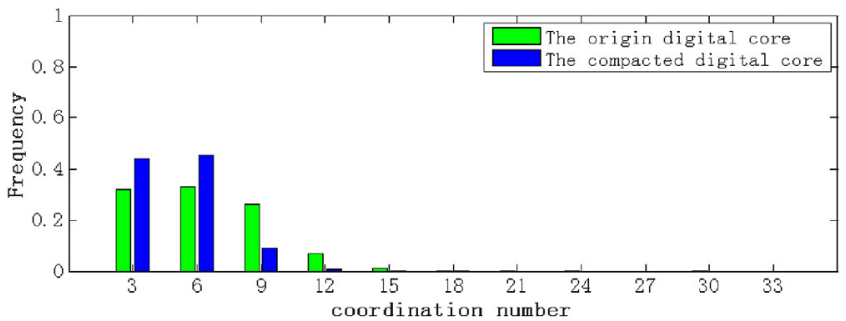

Type 1

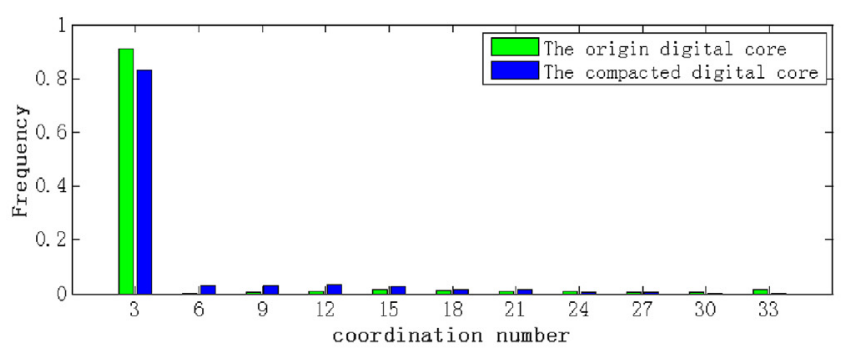

Type 3

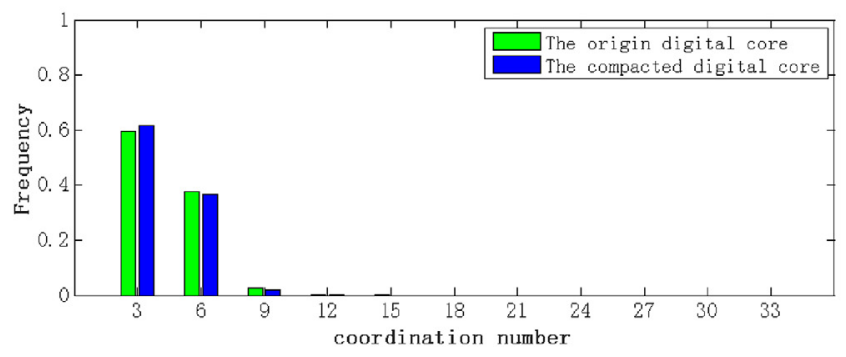

Type 5

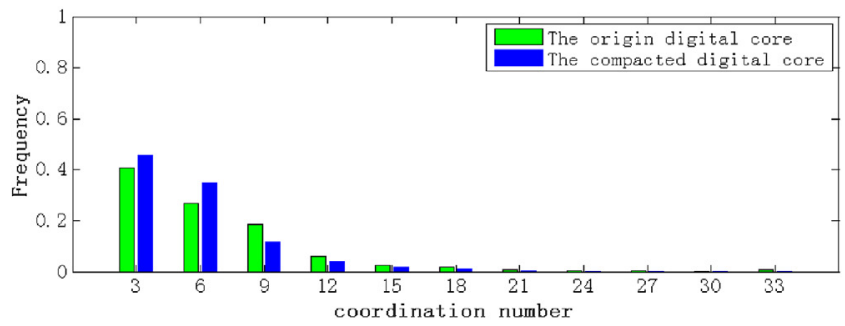

Type 2

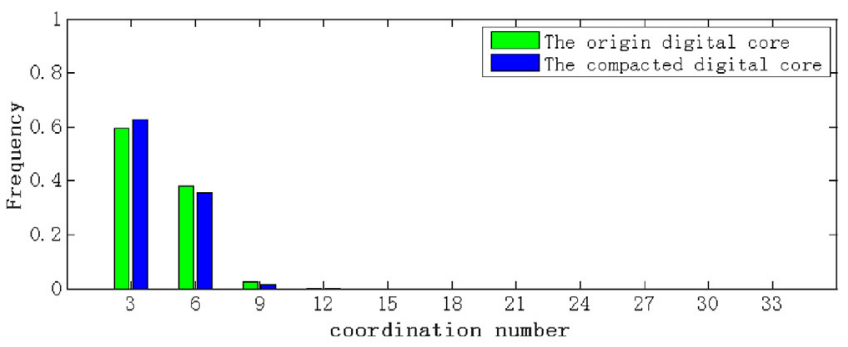

Type 4

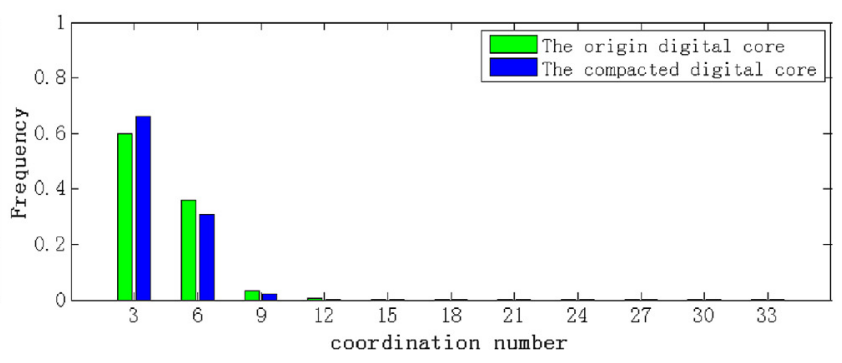

Type 6

Fig. 5. The coordination number distribution of each kind of typical organic pores before and after compaction.

The above six types of organic pores can represent the characteristics of most organic pores that exist in the marine Lower Silurian Longmaxi Formation in Changning-Weiyuan region of the Sichuan Basin. Studying the gas transport properties and gas-bearing property of OM with these typical organic pores will help us to evaluate the quality of the shale reservoir.

Moreover, to study the gas transport properties and gas-bearing property of OM with the above typical organic pores, we should determine the 3D structure of the organic pores. Here, we used a digital core reconstruction method, the CCISM-TSS method, to reconstruct the 3D structure of the organic pores. This method is a stochastic algorithm that integrates the advantage of the cross-correlation-based simulation method and a three-step sampling method (Ji et al., 2018). Especially, inspection the images in Fig. 1, it can be seen clearly that the distribution of organic pores is very heterogeneous. Therefore before reconstructing the digital core, we should obtain the RES (the representative elementary surface) for each image (Ji et al., 2019), as shown in Fig. 2. Inspecting Fig. 2, we can obtain that the sizes of RES for six types of organic pores are approximately $3.2 \mu \mathrm{m}, 3.2 \mu \mathrm{m}, 3.2 \mu \mathrm{m}$, $2.4 \mu \mathrm{m}, 0.6 \mu \mathrm{m}$ and $2.0 \mu \mathrm{m}$, respectively. Then we reconstructed the corresponding digital cores by the CCSIM-TSS method. The digital cores reconstructed the structure of each type of organic pores very well.

\subsection{The structures of typical organic pores under high temperature and pressure}

To study the gas transport propertie and gas-bearing property of $\mathrm{OM}$ with typical organic pores in a deep reservoir (under high temperature and pressure), we should first determine the 3D structure of the organic pores. In this paper, a digital core compressibility method is presented to obtain the 3D structure of organic pores in OM after compaction due to the high temperature and pressure in the deep reservoir.

\subsubsection{The digital core compressibility method}

The total pressure on a shale rock in a deep reservoir is the result of the coupled effects of the rock pressure caused by overburden and the interstitial fluid pressure in the rock. As the depth of the reservoir increases, the net load on the rock phase must increase, and the result is compaction of the rock structure, which produces a decrease in pore volume. Simultaneously, as the rock grains expand into the pore space, the fluid pressure increases. When the fluid pressure and the pressure due to overburden are equal, compaction of the rock stops.

Here we assume that because of the presence of pores in the rock, there are three volumes: the apparent volume of the rock $V_{b}$, the volume of the matrix $V_{s}$, and the volume of the pores $V_{p}$. Based on the theory of the rock compressibility, the relationship of the porosity of the rock and the effective stress satisfies:

$\varphi=\varphi_{0} \exp \left[-C_{p}\left(\sigma-p_{\text {in }}\right)\right]$

$\varphi_{0}=V_{p}^{0} / V_{b}^{0}, \varphi=V_{p}^{\prime} / V_{b}^{\prime}$

where $\varphi_{0}$ and $\varphi$ are the porosity of the rock at ambient temperature and pressure conditions and at the deep reservoir condition (high temperature and pressure). $C_{p}$ is the compression coefficient of organic matter (OM). $\sigma$ and $p_{\text {in }}$ are defined as the overburden pressure and the interstitial fluid pressure in the pores. $V_{p}^{0}$ and $V_{b}^{0}$ are the volume of 


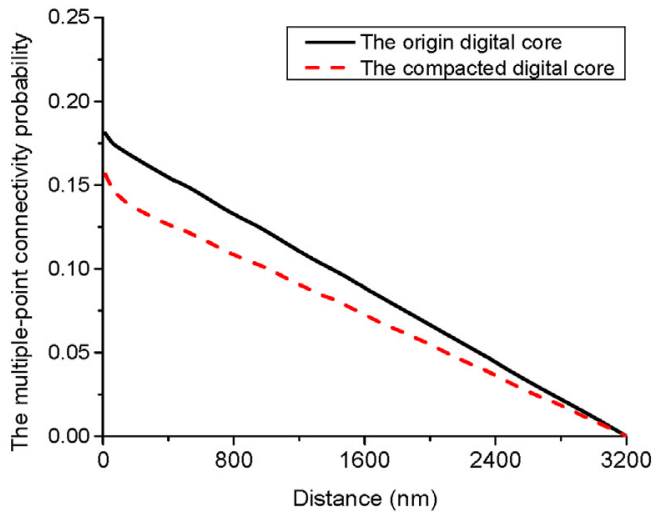

Type 1

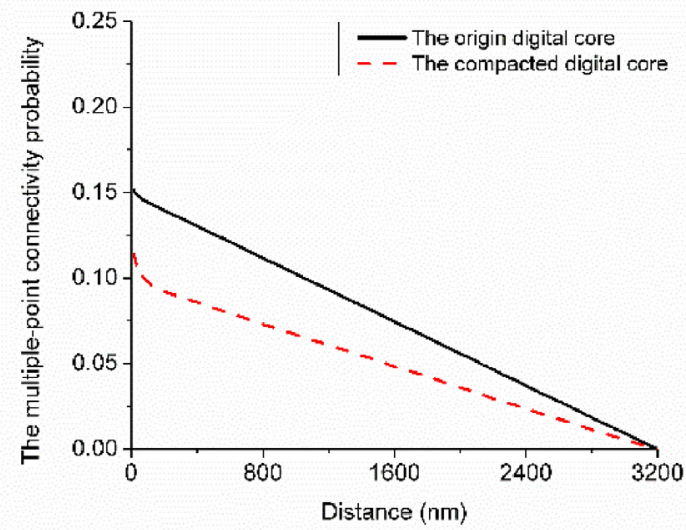

Type 3

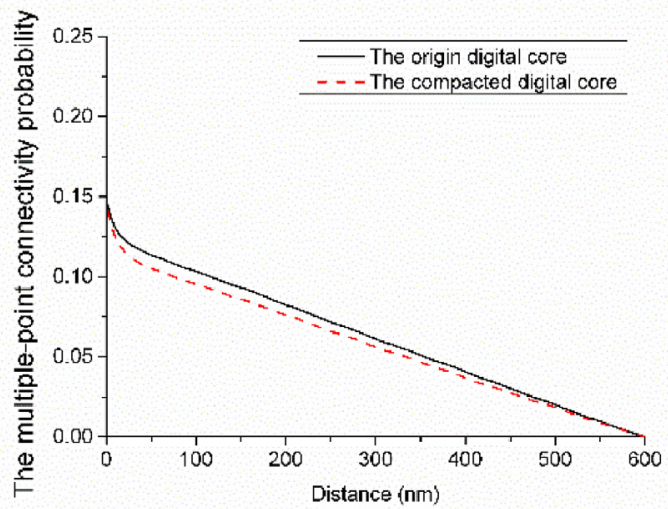

Type5

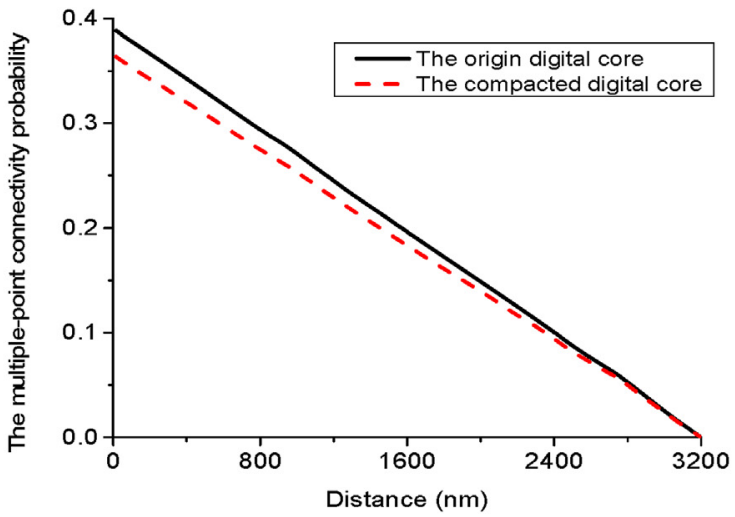

Type 2

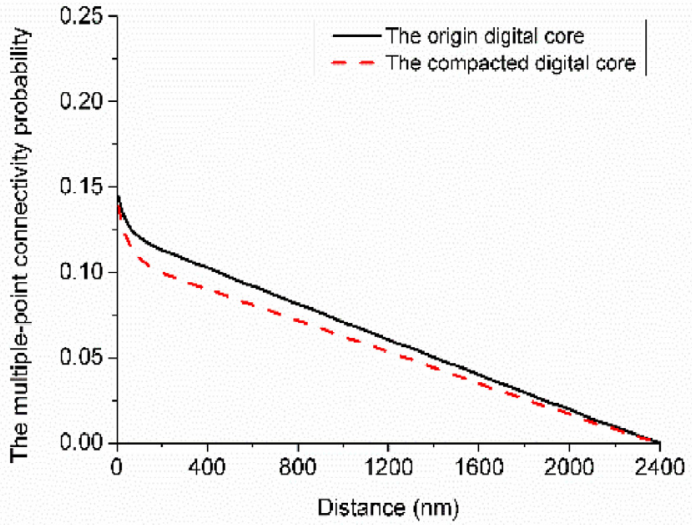

Type 4

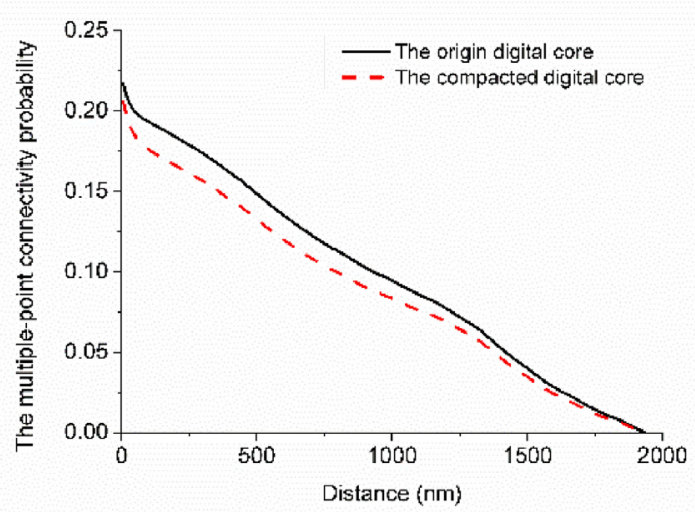

Type 6

Fig. 6. The multiple-point connectivity probability of each kind of typical organic pores before and after compaction. The distance in X-axis denotes the length of the connectivity path in the digital core.

pores and total volume of the shale rock at the reservoir condition (high temperature and pressure). $V_{p}^{\prime}$ and $V_{b}^{\prime}$ are the volume of pores and total volume of the shale rock at the reservoir condition (high temperature and pressure).

As mentioned above, as the depth of the reservoir increases, the overburden pressure must increase, and the rock grains expand into the pore space. The compaction of the pore space will induce the increase of fluid pressure. The fluid pressure and the volume of the pore should satisfy the equation of the state: $p_{\text {in }} V_{p}=n R T$

where $V_{p}$ and $p_{\text {in }}$ are defined as the volume of the pores in the rock and the pressure of the interstitial fluid in the pore; $n$ is the mole number of the gas in pores, $R$ is the gas constant $(8.31441 \mathrm{~J} / \mathrm{mol} / \mathrm{K})$, and $\mathrm{T}$ is the temperature.

We can solve the nonlinear equations Eqs. (1) and (3) by the Newton method and obtain the volume of the pores in the deep reservoir conditions (high temperature and pressure). Different from the 


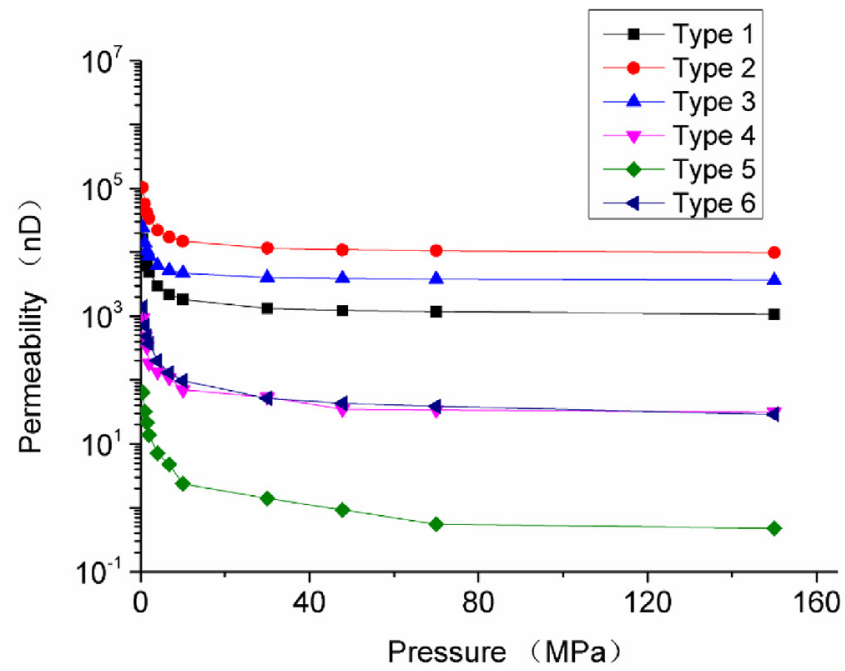

(a)

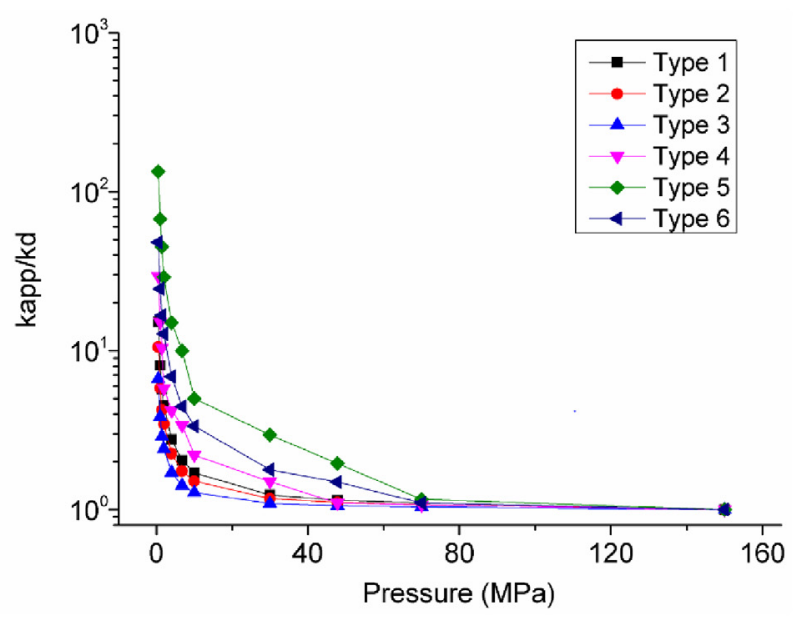

(b)

Fig. 7. The apparent permeability of OM with the six typical organic pores (a) and the ratio between the apparent and Darcy permeability at different pressures (b).

conventional method, our compressibility method can fully describe the coupling effect of the overburden and the interstitial fluid pressure during compaction. To examine the improved rock compressibility model, we calculate the relative change of the volume for different pores using the improved rock compressibility model and the conventional model without considering the variation of the fluid pressure in the pores. Here, the relative change of the volume is defined as the ratio of the volume change of the pore after and before compaction. The result is shown in Fig. 3. Inspecting this figure, we find that by considering the variation of the fluid pressure of the pore during compression, the improved rock compressibility model proposed in our paper can demonstrate the compaction difference between different pores. As the diameter of the pore increases, the relative change of volume during compression increases. Thus, we can deduce that small pores are more resistant to compaction than are big pores.

Based on the change of the volume for different pores defined in the above section, we can compact the digital core reconstructed by the CCSIM-TSS method. Considering that compaction is mainly caused by vertical effective stress due to overburden, we assume that the compaction of the digital core is a linear process by shifting the vertical coordinate of every mineral grain center according to the following formula:

$z^{\prime}=z_{0}(1-\lambda)$

where $z_{0}$ and $z^{\prime}$ are the vertical coordinates of the grain center before and after compaction, respectively; $\lambda$ is the compaction factor by which we can control the degree of compaction, and is obtained by the rock compressibility model mentioned above.

\subsubsection{Comparison of the structure for typical organic pores before and after compaction}

In this section, we investigate the 3D structure of typical organic pores under high temperature and pressure based on the digital core rock compressibility method presented in our paper.

The reservoir conditions discussed in this paper are as follows: the depth of the reservoir is $4000 \mathrm{~m}$, the temperature is $140{ }^{\circ} \mathrm{C}$, the overburden pressure $90.48 \mathrm{MPa}$, and the organic matter (OM) compressibility coefficient is $1.4 \times 10^{-3} \mathrm{MPa}^{-1}$. Under these conditions, we study the structure of the six kinds of typical organic pores.

First, to examine the structural change of the typical organic pores caused by the compaction effect under high temperature and pressure, we use the $A B$ (axis \& ball) algorithm (Yi et al., 2017), a newly developed pore network extraction algorithm, to extract the pore networks from the digital cores, and then calculate the pore radius and coordination number. The pore-size distributions of the six types of organic pores before and after compaction are shown in Fig. 4. After compacted, the proportion of some small pores in OM increases, whereas the proportion of big pores decreases. To further demonstrate the connectivity of the six types of organic pores after compaction, Figs. 5 and 6 also plot the coordination number distributions and the multiple-point connectivity probabilities of six types of organic pores before and after compaction. After compaction, the coordination number decreases, and the connectivity of the typical organic pores decreases.

\subsection{Apparent permeability in organic pores}

In this section, we study the apparent permeability in OM with typical organic pores using the methodology described in the above section.

\subsection{The apparent permeability in typical organic pores}

To investigate the gas transport propertie of OM with typical organic pores, the apparent permeability of OM is calculated, as shown in Fig. 7. It should be pointed out that the apparent permeability in this paper was calculated using the model developed by Jiang and Lin (2017), which can account for the slippage effect and the Knudsen diffusion of viscous flow in the organic pores. As shown in Fig. 7a, OM filled with Type 2 organic pores has the greatest permeability, OM filled with Type 1 and 3 organic pores has much greater permeability than that filled with Types 4, 5, and 6 organic pores. OM filled with Type 5 organic pordes has the lowest permeability. To investigate the nonDarcy effect of each type of organic pores, Fig. 7b also plots the ratio between the apparent and Darcy permeability (kapp and kd). It can be seen that in contrast with the apparent permeability result, OM filled with Type 5 organic pores has the largest value of kapp/kd, whereas OM with Type 3 organic pores has the smallest value of kapp/kd. This result is reasonable, because Type 5 organic pores have the smallest size among the six typical organic pores.

\subsection{The regularities of the apparent permeability in organic pores}

In this section, we focus on determining the regularity of the gas transport properties for OM with different pores. Because the six kinds of typical organic pores have quite different pore-size distributions, it is 


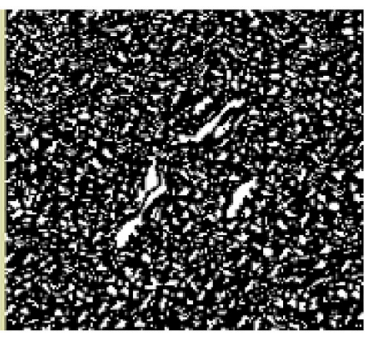

MAE1

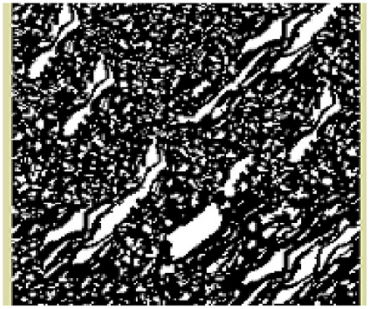

MAE5

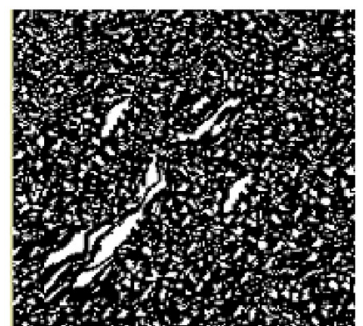

MAE2

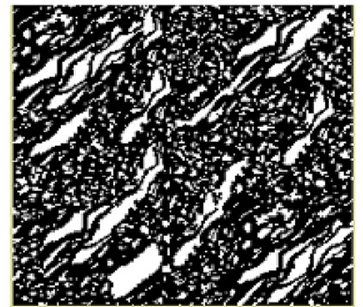

MAE6

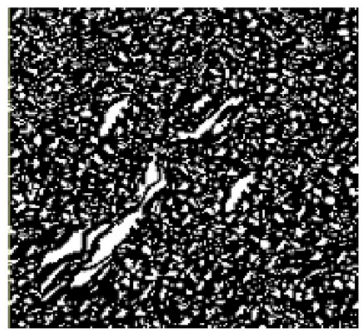

MAE3

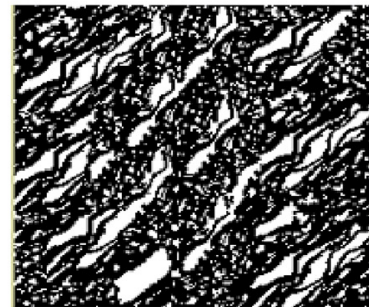

MAE7

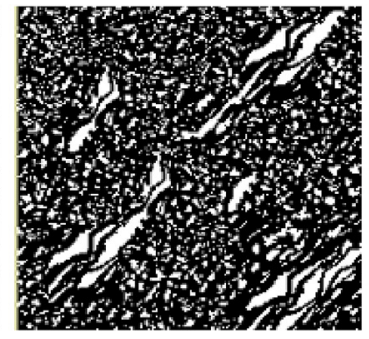

MAE4

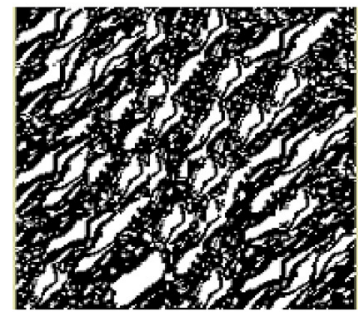

MAE8

Fig. 8. Images of the eight models.

Table 2

The proportions of macropores and mesopores for the eight models.

\begin{tabular}{lllllllll}
\hline & MAE1 & MAE2 & MAE3 & MAE4 & MAE5 & MAE6 & MAE7 & MAE8 \\
\hline Macropores & $10 \%$ & $20 \%$ & $30 \%$ & $40 \%$ & $50 \%$ & $60 \%$ & $70 \%$ & $80 \%$ \\
Mesopores & $90 \%$ & $80 \%$ & $70 \%$ & $60 \%$ & $50 \%$ & $40 \%$ & $30 \%$ & $20 \%$
\end{tabular}

difficult to determine the regularity of the gas transport propertie directly based on the six kinds of organic pores. Thus, we generated some models based on Type 1 organic pores to determine the regularity of the flow mechanism in OM.

First, we set the pore-size distribution of mesopores as fixed, and then increased the proportion of macropores. During this process, the pore-size distribution of macropores was also unchanged. Based on the above rule, we generated eight models, defined as MAE1, MAE2, MAE3, MAE4, MAE5, MAE6, MAE7, and MAE8, as shown in Fig. 8. The proportions of macropores and mesopores are listed in Table 2. It should be emphasized that the biggest difference among the eight models was the proportion of macropores and mesopores. Thus, we investigated the regularity of the flow mechanism in OM based on these models.

The apparent permeabilities for the eight models were calculated. To summarize the regularity of the flow, here we chose the Darcy permeability for eight models to plot, as shown in Fig. 9a. Moreover, to

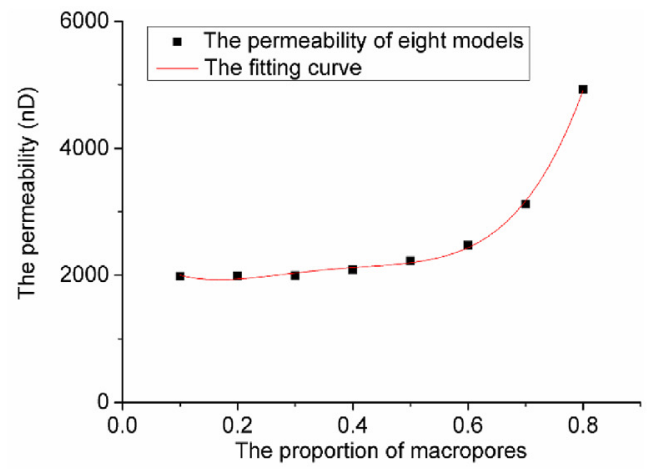

(a) investigate the non-Darcy effect, we also plotted the ratio of the apparent and Darcy permeabilities for the eight models, as shown in Fig. 9b. Note that the X-axes of Fig. 9a and b represent the proportion of macropores in the eight models. It is clear that the permeability increased with the proportion of macropores in the models, and there were positive correlations between the permeability and the biquadratic of the proportion of macropores. However, the value of kapp/kd decreased with the proportion of macropores in the models, and there were negative correlations between the value of kapp/kd and the cubic function of the proportion of macropores.

The above models are composed of macropores and mesopores. Next, we investigated models with micropores and mesopores. Like the above models, we set the pore-size distribution of micropores as fixed, and then increased the proportion of mesopores. During this process, the pore-size distribution of mesopores was also unchanged. Based on the above rule, we generated eight models, defined as MEI 1, MEI 2, MEI 3, MEI 4, MEI 5, MEI 6, MEI 7, and MEI 8, as shown in Fig. 10. The proportions of micropores and mesopores are listed in Table 3, and the proportion of mesopores increases from MEI 1 to MEI 8.

Similarly, the apparent permeabilities for the eight models were calculated. The Darcy permeabilities and the ratio of the apparent and Darcy permeabilities for the eight models are plotted in Fig. 11. Also, the X-axes of the two figures indicate the proportion of mesopores in the eight models. Like the results of the MAE models, the permeability

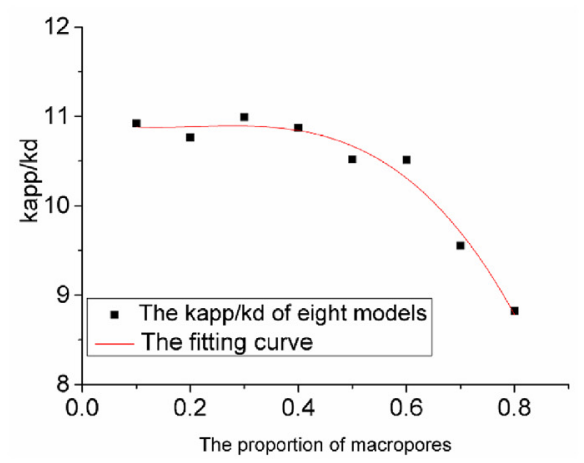

(b)

Fig. 9. The permeability (b) and the ratio of kapp and kd (b) of the eight models. 


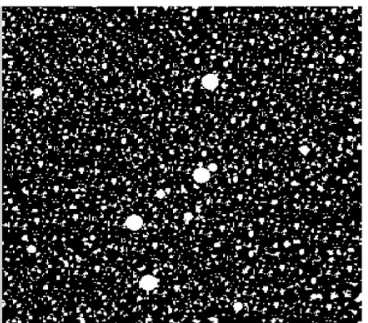

MEI 1

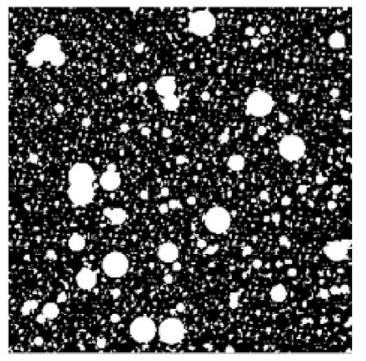

MEI 5

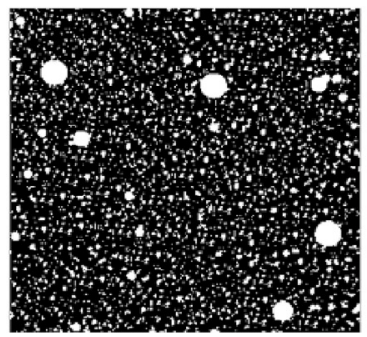

MEI 2

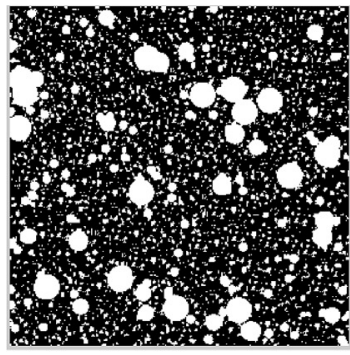

MEI 6

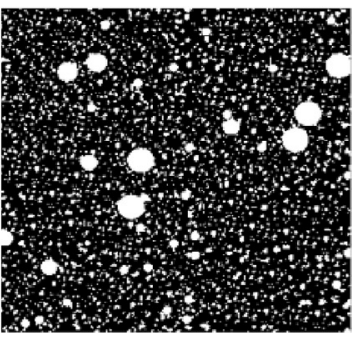

MEI 3

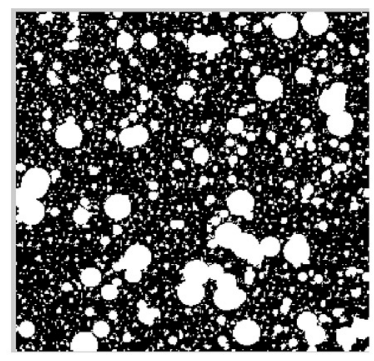

MEI 37

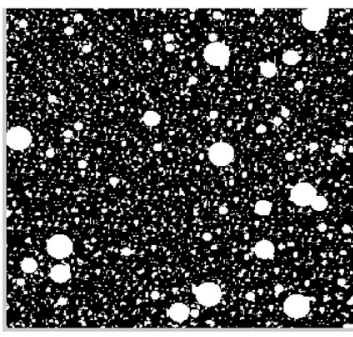

MEI 4

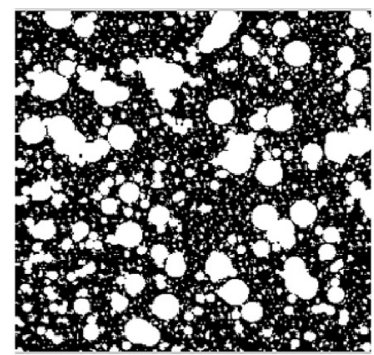

MEI 8

Fig. 10. Images of the eight models.

Table 3

The proportions of micropores and mesopores for eight models.

\begin{tabular}{lcccccccc}
\hline & MEI 1 & MEI 2 & MEI 3 & MEI 4 & MEI 5 & MEI 6 & MEI 7 & MEI 8 \\
\hline Mesopores & $10 \%$ & $20 \%$ & $30 \%$ & $40 \%$ & $50 \%$ & $60 \%$ & $70 \%$ & $80 \%$ \\
Micropores & $90 \%$ & $80 \%$ & $70 \%$ & $60 \%$ & $50 \%$ & $40 \%$ & $30 \%$ & $20 \%$ \\
\hline
\end{tabular}

increased with the proportion of mesopores in the models. Positive correlations between the permeability and the square of the proportion of mesopores were also obtained by curve fitting. Negative correlations between the value of $\mathrm{kapp} / \mathrm{kd}$ and the proportion of mesopores were clearly observed. Moreover, the fitting results for the MEI models were quite different from those of the MAE models.

Based on the above analysis of the MAE and MEI models, the relationship between permeability and the proportions of micropores, mesopores, and macropores can be further determined. Finally, a chart of the gas transport propertie in the OM can be obtained, shown in Fig. 12. Based on this figure, we can quickly judge the non-Darcy effect of OM.

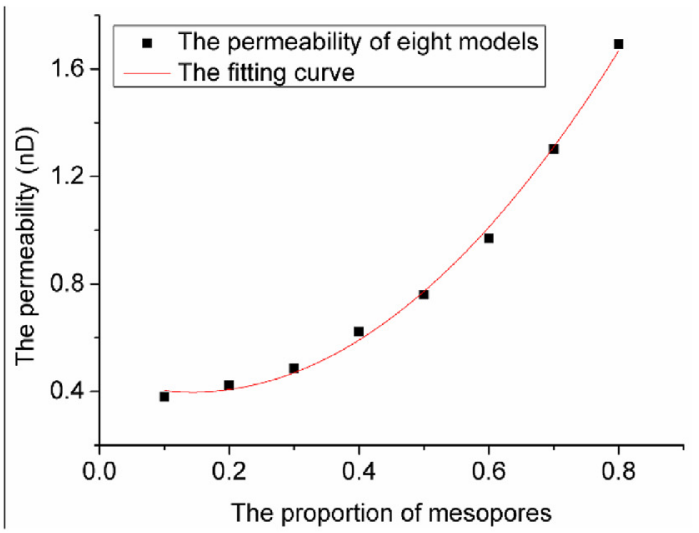

(a)
2.6. Apparent permeability in typical organic pores under high temperature and pressure

For this section, we investigated the apparent permeability of OM with the typical organic pores under high temperature and pressure.

First, to examine the apparent permeability of gas in organic pores under deep reservoir conditions, we calculated the permeability for $\mathrm{OM}$ with the organic pores at different depths in the reservoir $(1000 \mathrm{~m}$, $2000 \mathrm{~m}, 3000 \mathrm{~m}$, and $4000 \mathrm{~m}$ ), as shown in Fig. 13. The results show that as the depth of the reservoir increases, the permeability of OM with the typical organic pores decreases correspondingly.

Furthermore, we defined the relative change of the permeability of the compacted $\mathrm{OM}$ as Pr. Fig. 14 depicts the relative change of the permeability of OM after compaction at the depth of $4000 \mathrm{~m}$. We determined that the permeability of OM with Type 1 organic pores had the biggest change $(42.16 \%)$, whereas the OM with Type 5 organic pores had the smallest change (22.3\%). This is because Type 1 organic pores have a large proportion of strip-shaped macropores, which can be compacted more easily. In contrast, there are more micropores in Type 5 organic pores, which are the most difficult to compact. The

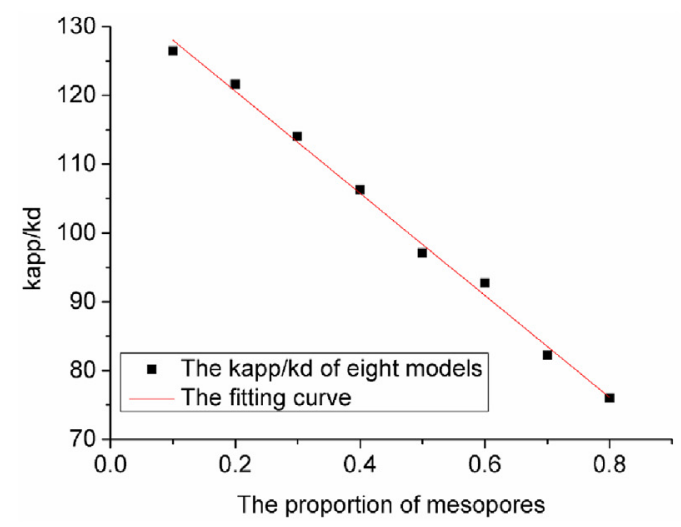

Fig. 11. The permeability (a) and the ratio of kapp and kd (b) of the eight models. 


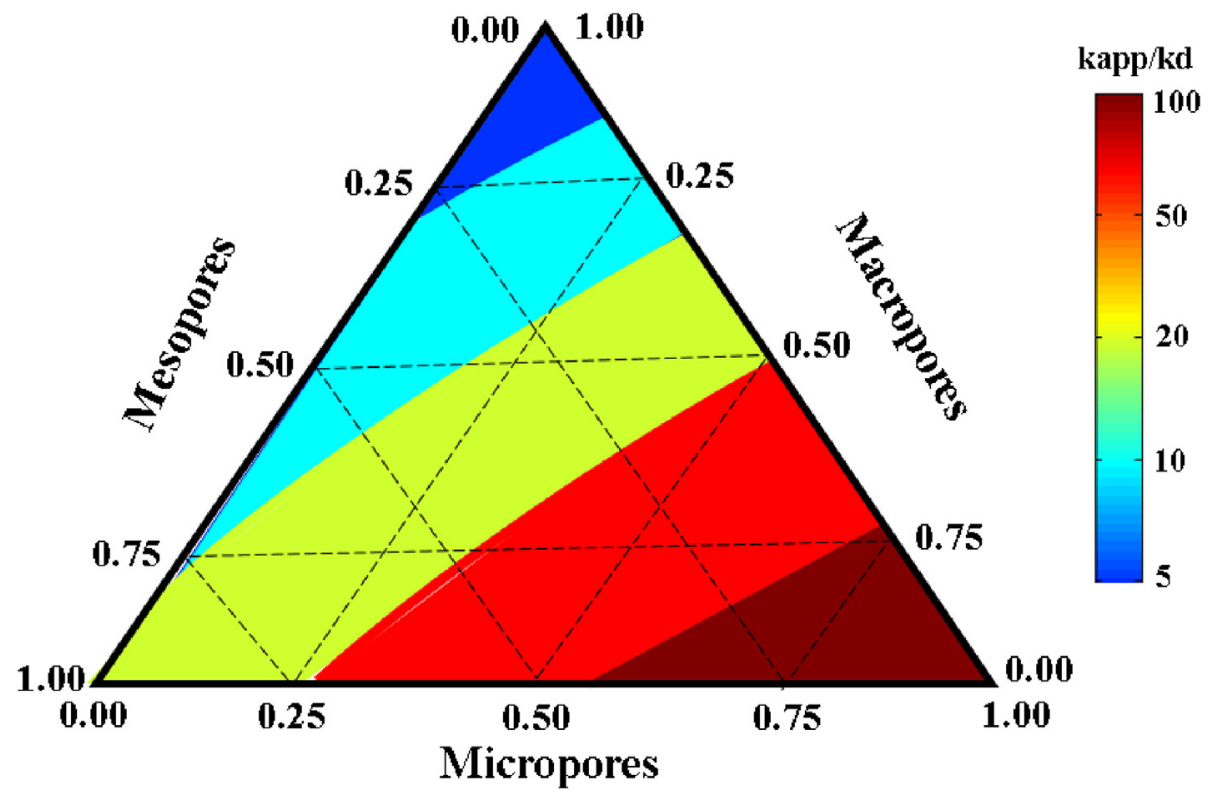

Fig. 12. The chart of the gas transport propertie in OM.

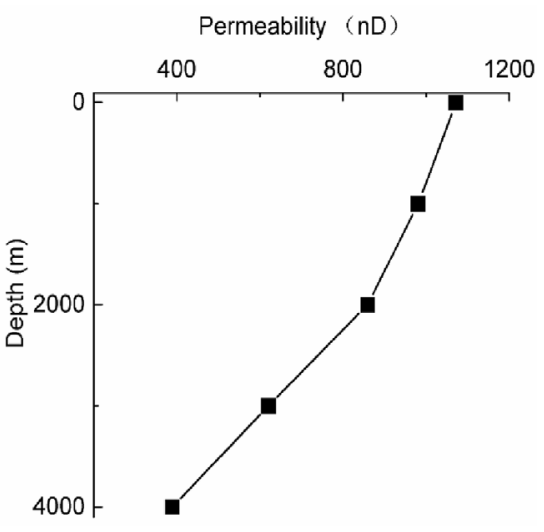

Type 1

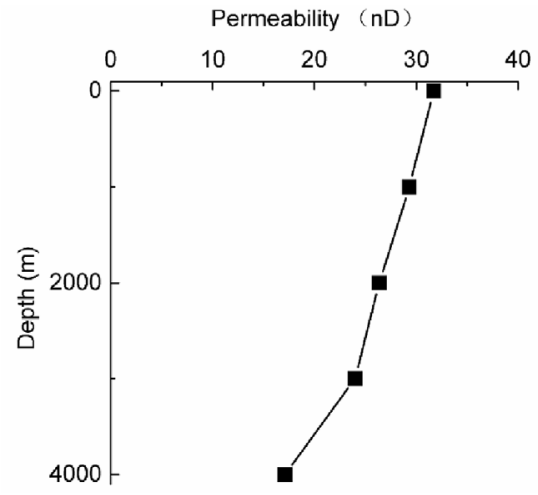

Type 4

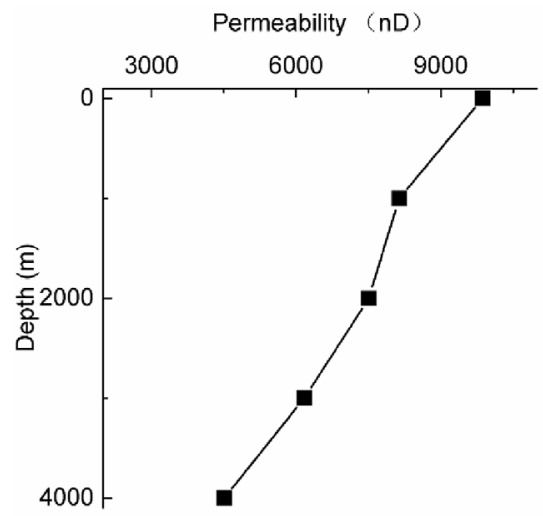

Type 2

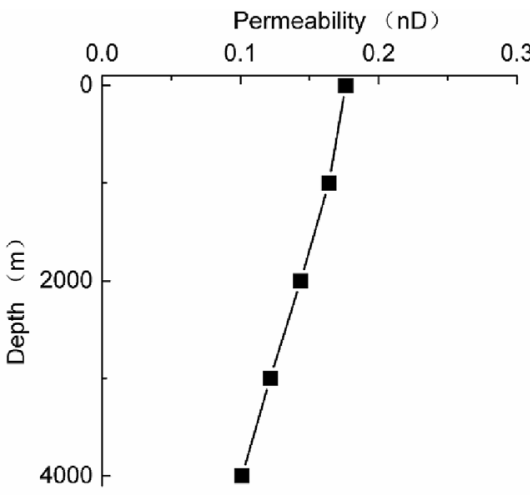

Type5

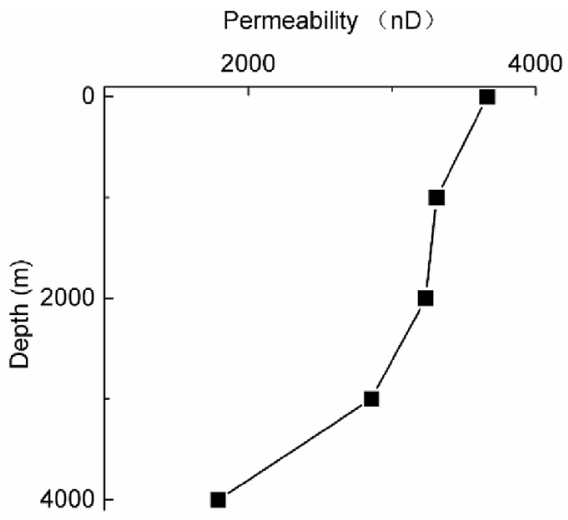

Type 3

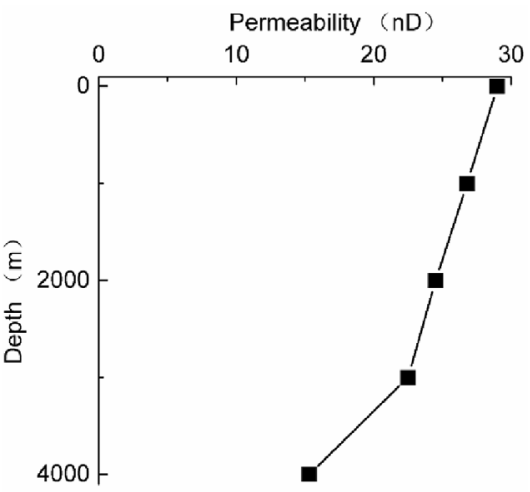

Type 6

Fig. 13. The permeability of OM with the typical organic pores at different depths of the reservoir.

distributions of the typical organic pores before and after compaction in the chart of the apparent permeability are compared in Fig. 16.

\section{Gas-bearing property of $\mathrm{OM}$}

In this section, we investigate the gas-bearing property of OM with the typical organic pores using the methodology described in section 2 .

3.1. The gas-bearing property of OM with the typical organic pores

To investigate the gas-bearing property of OM with the typical organic pores, the absolute adsorption amount and the free gas amount of 


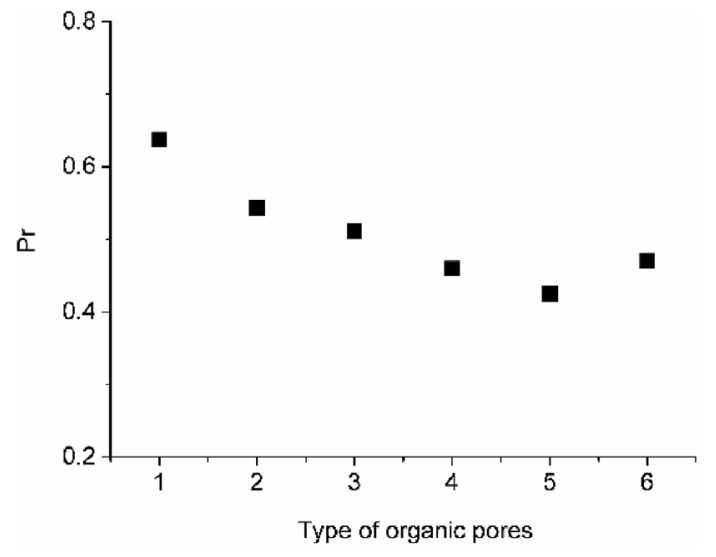

Fig. 14. Relative change of the permeability of the six typical organic pores after compaction at the depth of $4000 \mathrm{~m}$.

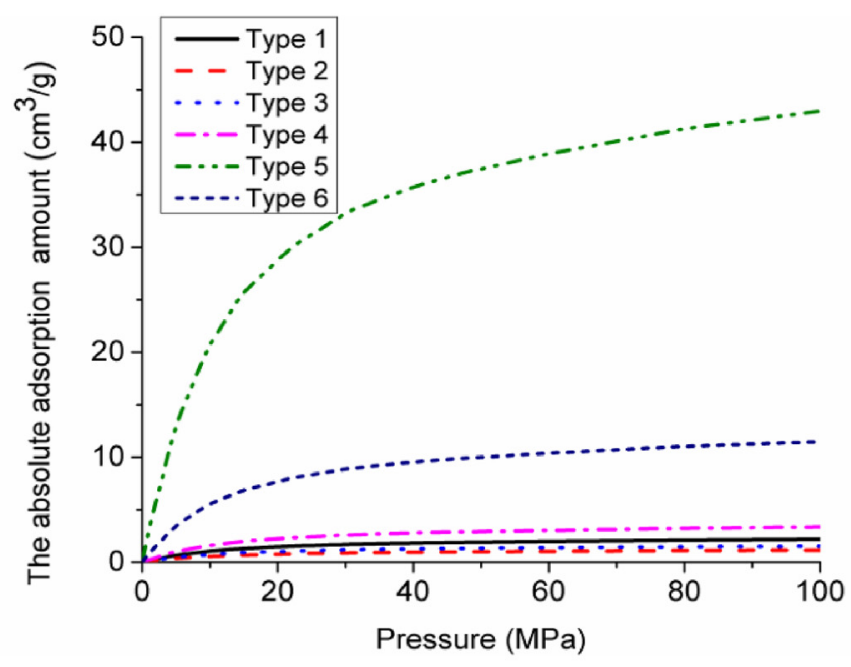

(a)

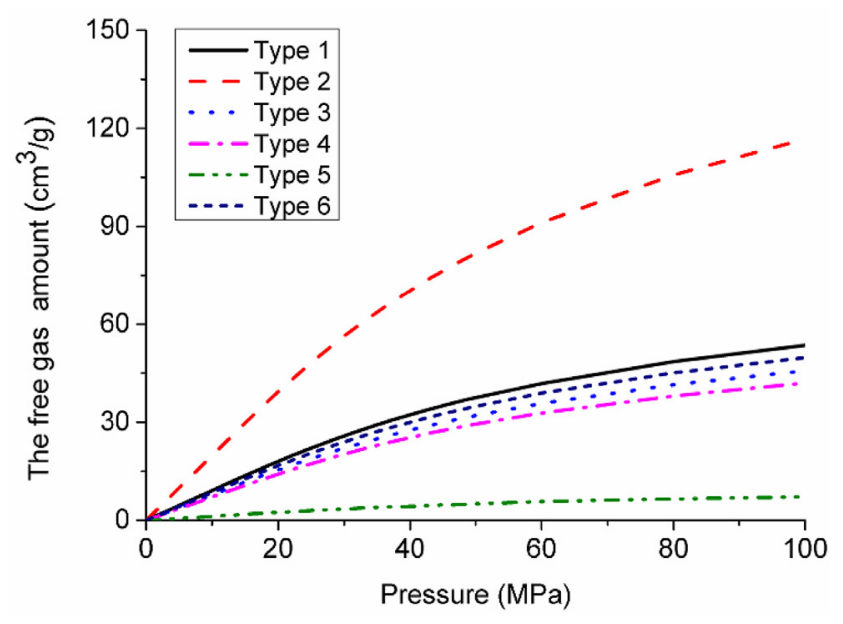

(b)

Fig. 15. The absolute adsorption amount and the free gas amount of OM with the typical organic pores at different pressures.

OM with the typical organic pores were calculated, as shown in Fig. 15. It should be pointed out that the absolute adsorption amount in this paper was calculated based on the molecular dynamics (MD) model developed by Jiang and Lin (2018). Fig. 15a shows that OM filled with
Type 5 organic pores had the largest absolute adsorption amount, and that OM filled with Type 6 organic pores had a larger absolute adsorption amount than that filled with Types 1, 2, 3, and 4 organic pores. OM filled with Type 2 organic pores had the smallest absolute adsorption amount. Fig. 15b plots the variance of the free gas amount with pressure. In contrast with the absolute adsorption amount results, OM filled with Type 2 organic pores had the largest free gas amount, and OM with Type 5 organic pores had the smallest free gas amount. These results are reasonable, because Type 5 organic pores have the smallest size among the six typical organic pores, and Type 2 organic pores have the largest proportion of macropores among the six typical organic pores. We also found that for each type of organic pores, as pressure increased, the absolute adsorption amount and the free gas amount increased correspondingly.

\subsection{The gas-bearing property of OM under high temperature and pressure}

To examine the gas-bearing property of the organic pores under deep reservoir conditions, we calculated the absolute adsorption amount and the free gas amount for OM with organic pores at different depths of the reservoir $(1000 \mathrm{~m}, 2000 \mathrm{~m}, 3000 \mathrm{~m}, 4000 \mathrm{~m})$, as shown in Fig. 16. Here, we chose Type 1 and Type 5 organic pores to study the gas-bearing property under deep reservoir conditions. The free gas amount of OM filled with Type 1 organic pores was much bigger than the absolute adsorption amount, whereas the free gas amount of OM filled with Type 5 organic pores was much smaller than the absolute adsorption amount. The reason is that Type 1 organic pores have a large proportion of macropores and a small proportion of micropores; thus, the porosity is high and the specific surface area is small. Correspondingly, Type 5 organic pores have a large portion of micropores and a small portion of mesopores; thus, the porosity is relatively small and the specific surface area is large. Fig. 16 also shows that the absolute adsorption amount of OM filled with Type 1 and 5 organic pores first increased with the depth and then decreased with depth. However, the free adsorption amount of OM filled with Type 1 and 5 organic pores increased with the depth of the reservoir. This result is reasonable because under low temperature and pressure, the absolute adsorption amount increases quickly with pressure and temperature, and as the pressure increases, the absolute adsorption amount increases slowly ( $\mathrm{Lu}$ et al., 1995; Jiang and Lin, 2018). At the same time, as depth increases, compaction of the organic pores increases. The absolute adsorption amount in organic pores is the result of the coupled effects of compaction, temperature, and pressure. At lower depths, the increased velocity of the absolute adsorption amount due to the temperature and pressure is greater than the compaction; thus, the absolute adsorption amount increases at first. When it becomes smaller than the compaction, the absolute adsorption amount then decreases with depth. Correspondingly, the increased velocity of the free gas amount due to the temperature and pressure is always greater than the compaction; thus, the free adsorption amount of OM with typical organic pores increases with the depth of the reservoir.

Next, we focused on determining the regularity of the gas-bearing property for OM with different types of pores. Similar to the method described in the above section, we used the models MAE 1, MAE 2, MAE 3, MAE 4, MAE 5, MAE 6, MAE 7, MAE 8, MEI 1, MEI 2, MEI 3, MEI 4, MEI 5, MEI 6, MEI 7, and MEI 8 as shown in Figs. 7 and 8 to study the gas-bearing property for $\mathrm{OM}$ with different structures of pores. It should be pointed out that under conventional temperature $\left(20^{\circ} \mathrm{C}\right)$ and pressure $(0.1 \mathrm{MPa})$, the absolute adsorption amount and the free gas amount are very small, as shown in Fig. 15. It is meaningless to study the gas-bearing property under conventional temperature and pressure. Thus, we focused on the gas-bearing property under high temperature and pressure. Fig. 17 plots the absolute adsorption amount and the free gas amount for eight models, MAE 1, MAE 2, MAE 3, MAE 4, MAE 5, MAE 6, MAE 7, and MAE 8, at the depths of $1000 \mathrm{~m}$ and $4000 \mathrm{~m}$. The results reveal that at the same depth, the absolute adsorption amount 


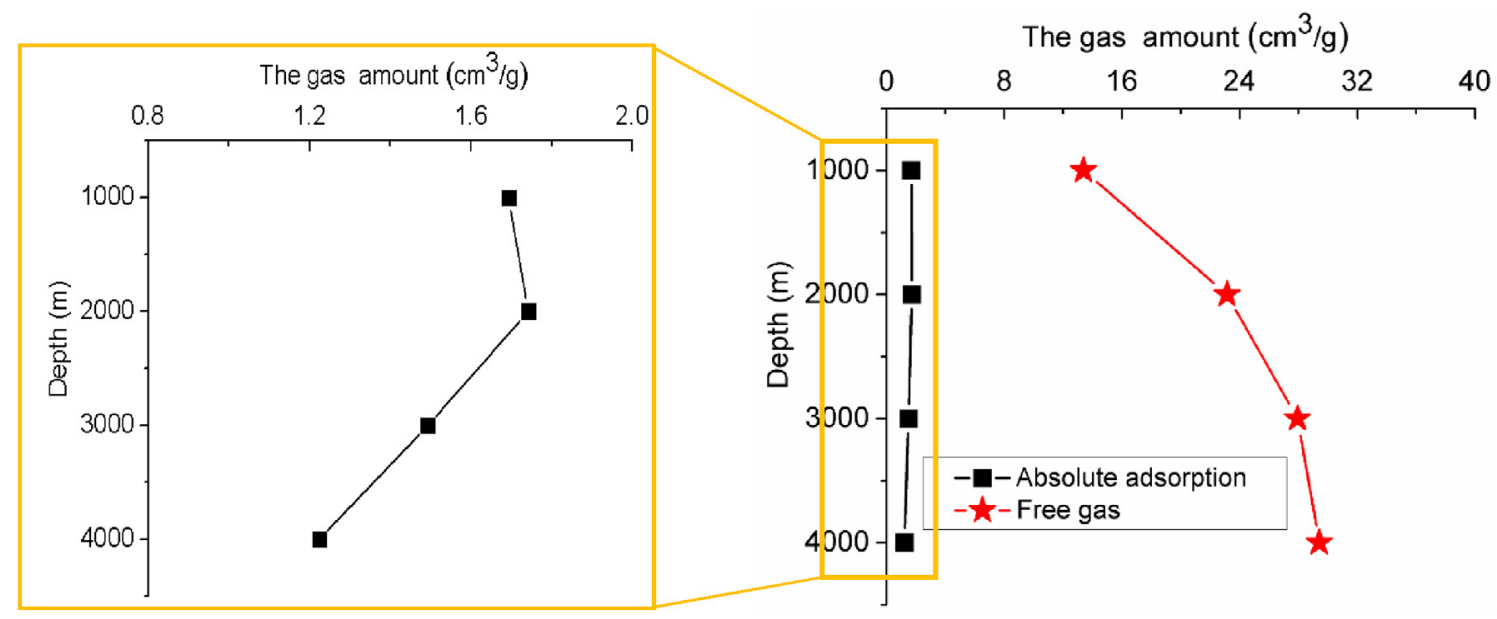

(a)

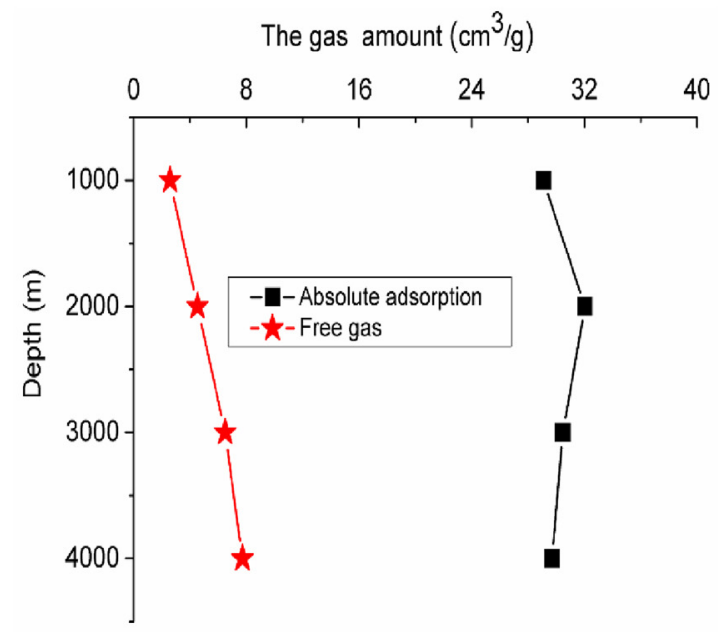

(b)

Fig. 16. The absolute adsorption amount and the free gas amount of OM with the Type 1 (a) and Type 5 (b) organic pores at different depth.

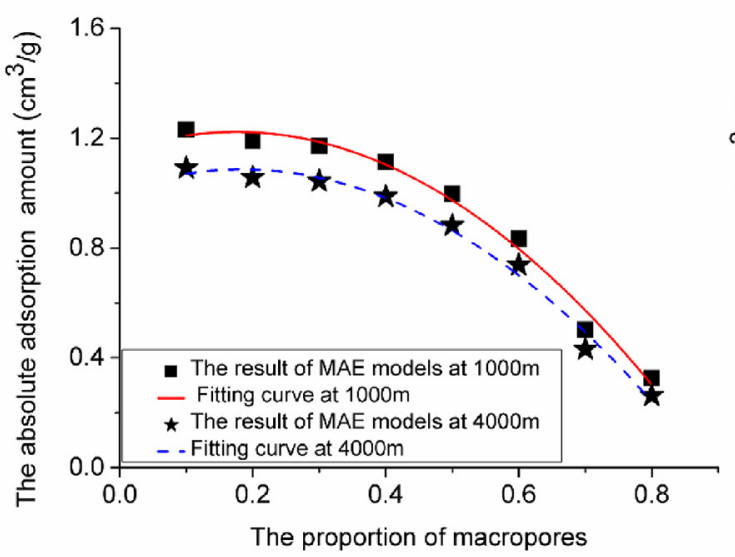

(a)

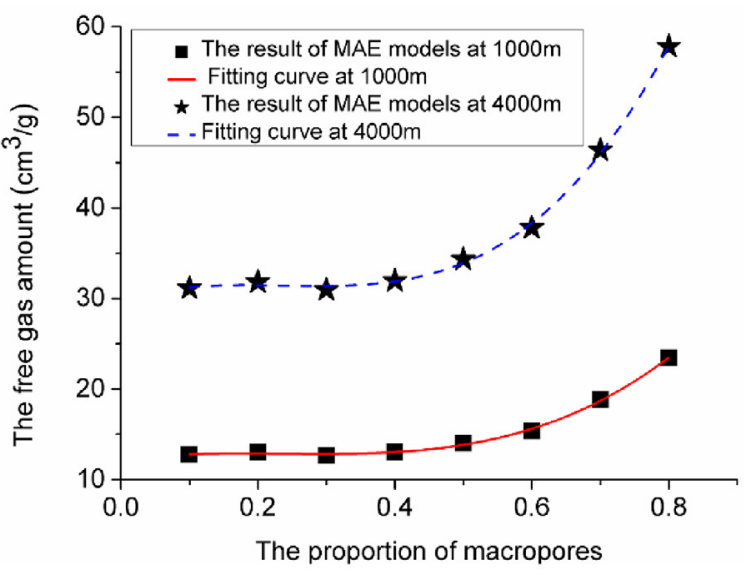

(b)

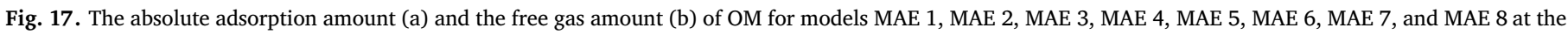
depths of $1000 \mathrm{~m}$ and $4000 \mathrm{~m}$. 


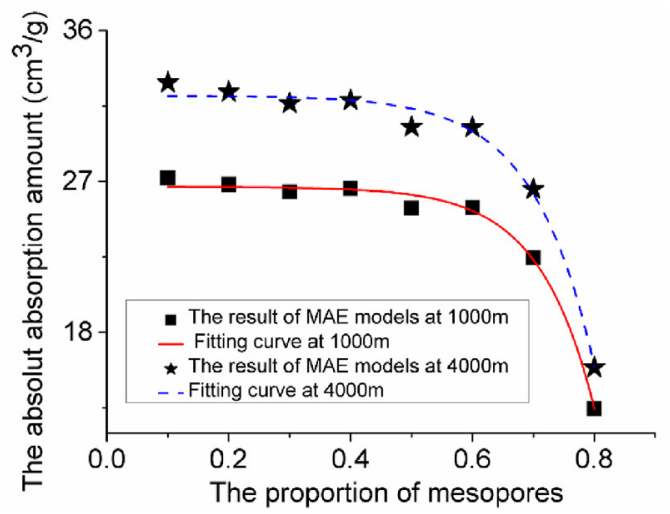

(a)The absolute adsorption amount of OM

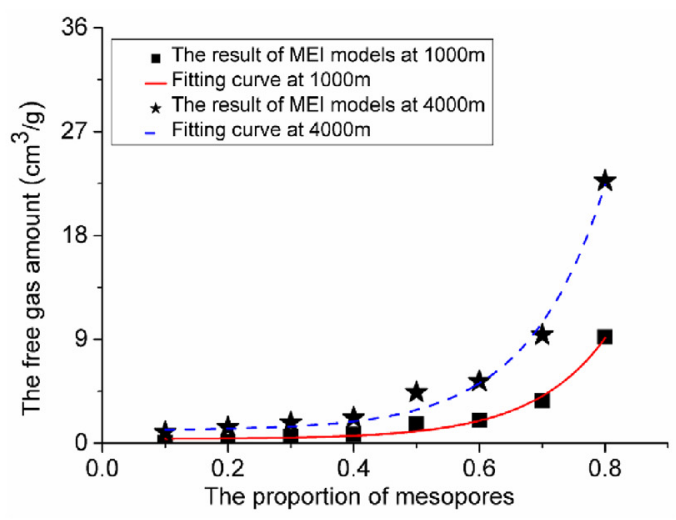

(b) The free gas amount of $\mathrm{OM}$

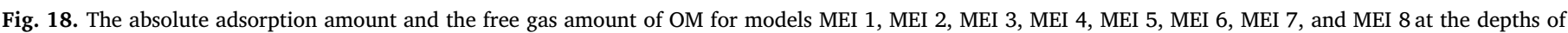
$1000 \mathrm{~m}$ and $4000 \mathrm{~m}$, (a)The absolute adsorption amount of OM, (b) The free gas amount of OM.

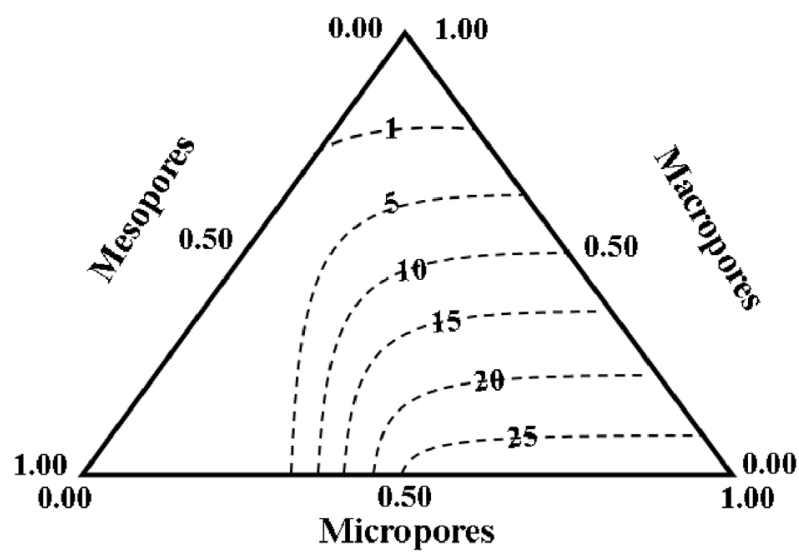

(a)The absolute adsorption amount of $\mathrm{OM}$

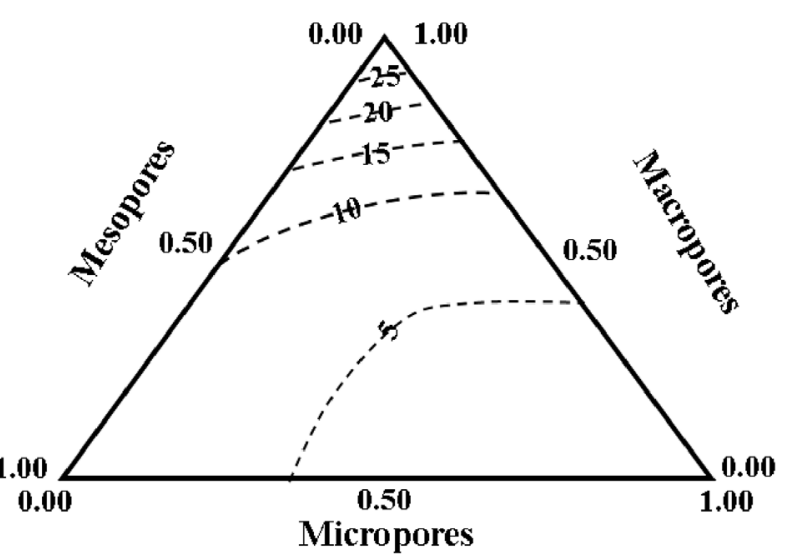

(b) The free gas amount of OM

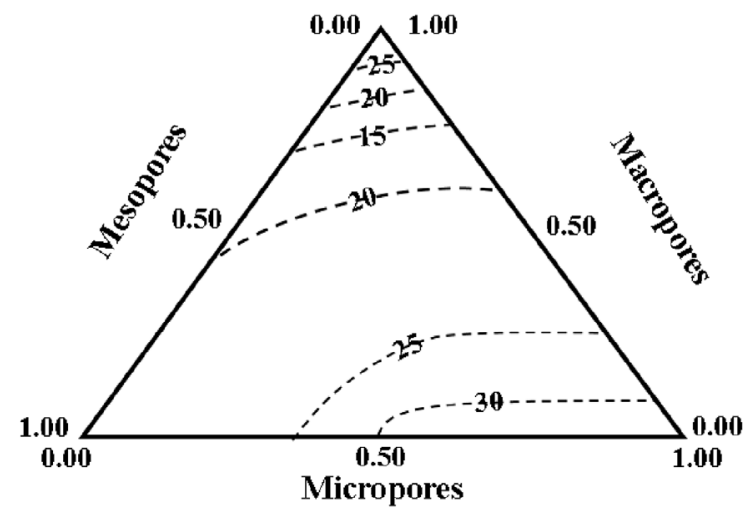

(c) The total gas amount of $\mathrm{OM}$

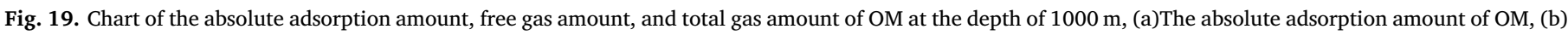
The free gas amount of OM, (c) The total gas amount of OM.

decreases with the proportion of macropores in the models, whereas the free gas amount increases with the proportion of macropores in the models. After fitting the curve, we found that the absolute adsorption amount is a quadratic function of the proportion of macropores, and the free gas amount is a cubic function of the proportion of macropores. Interestingly, we also found that for the same model, the absolute adsorption amount at $4000 \mathrm{~m}$ is smaller than that at $1000 \mathrm{~m}$, whereas the free gas amount at $4000 \mathrm{~m}$ is larger than that at $1000 \mathrm{~m}$.

Moreover, the absolute adsorption amount and the free gas amount for the eight models MEI 1, MEI 2, MEI 3, MEI 4, MEI 5, MEI 6, MEI 7, and MEI 8 at the depths of $1000 \mathrm{~m}$ and $4000 \mathrm{~m}$ were also calculated, as shown in Fig. 18. The absolute adsorption amount decreased with the 


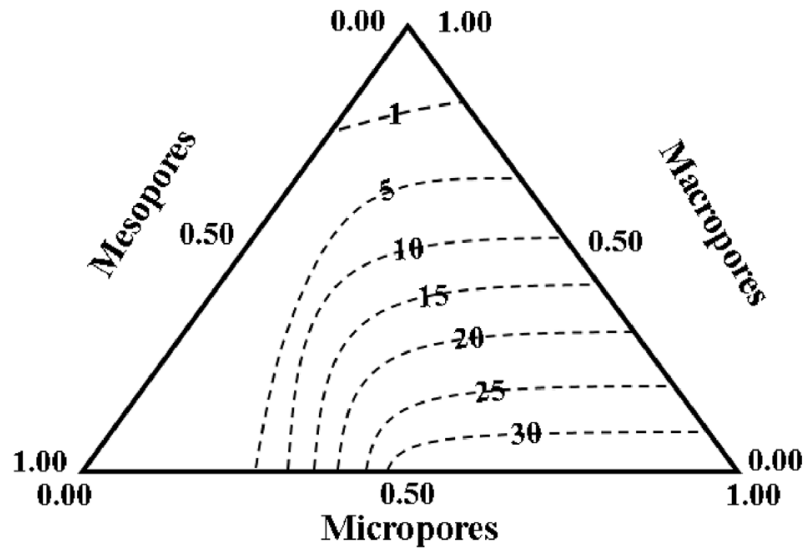

(a)The absolute adsorption amount of $\mathrm{OM}$

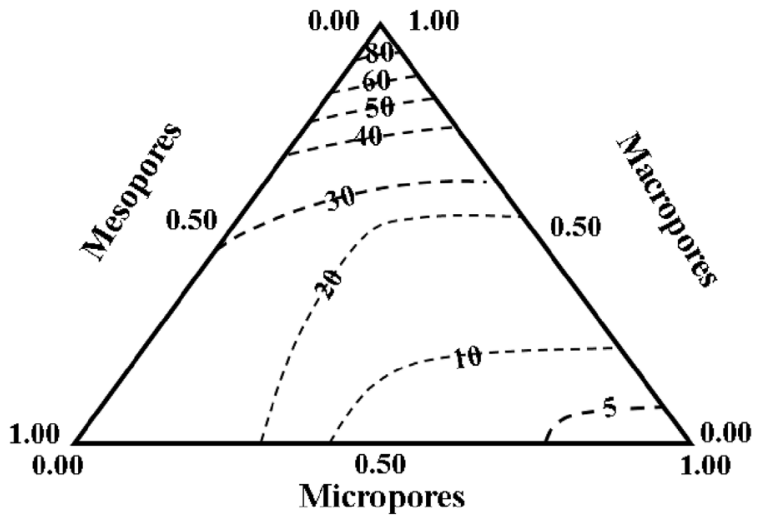

(b) The free gas amount of $\mathrm{OM}$

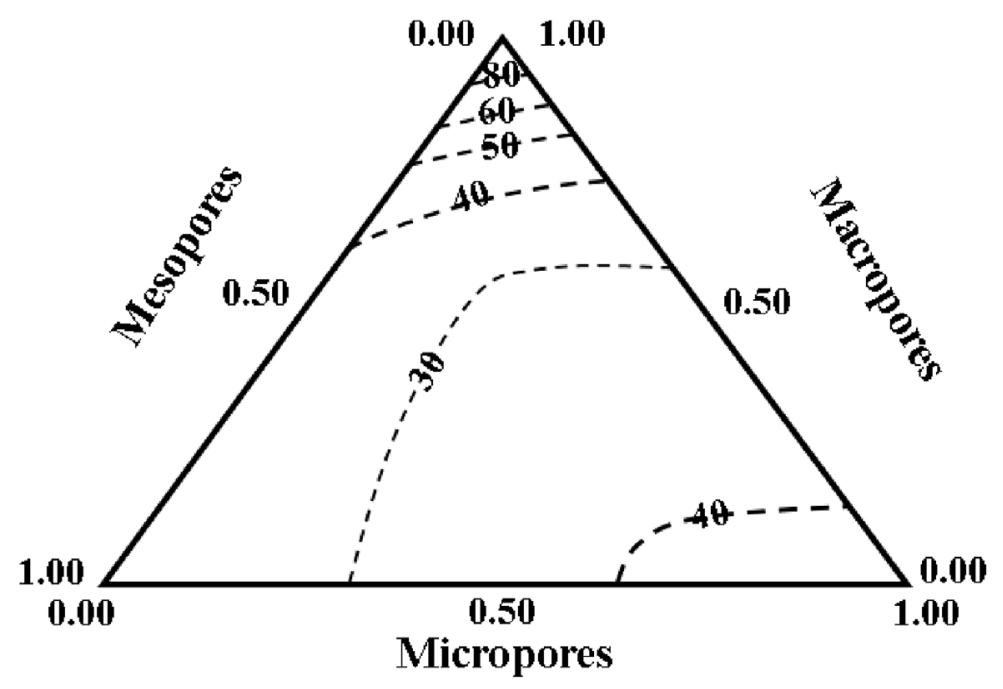

(c) The total gas amount of OM

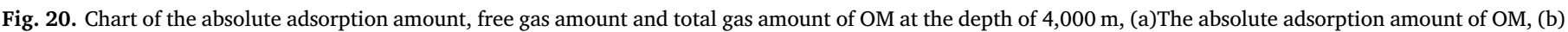
The free gas amount of OM, (c) The total gas amount of OM.

proportion of mesopores in the models, whereas the free gas amount increased with the proportion of mesopores in the models. Fitting the curve, we determined that the absolute adsorption amount is an exponential function of the proportion of mesopores, and the free gas amount is also an exponential function of the proportion of mesopores. These results demonstrate that as the proportion of mesopores increases, the difference of the free gas amount between $1000 \mathrm{~m}$ and $4000 \mathrm{~m}$ increases, and the difference of the absolute adsorption amount between $1000 \mathrm{~m}$ and $4000 \mathrm{~m}$ decreases. We also find that for the same model, the free gas amount at $4000 \mathrm{~m}$ is larger than that at $1000 \mathrm{~m}$, and this result is the same as that for the MAE models. The absolute adsorption amount at $4000 \mathrm{~m}$ is larger than that at $1000 \mathrm{~m}$, and this result is different from that of the MAE models. This is because the pore size of the MEI models is smaller than that of the MAE models, and the compaction effect is smaller than that of the MAE models. In addition, the absolute adsorption amount of the MEI models is much bigger than that of the MAE models, so the increment velocity of the absolute adsorption amount due to the temperature and pressure is always greater than the compaction effect for MEI.

Based on the analysis of the MAE and MEI models, the relationships between the absolute adsorption amount, the free gas amount, and the proportions of micropores, mesopores and macropores can be further determined. Then, the chart of the absolute adsorption amount, the free gas amount, and the total gas amount at the depths of 1000 and $4000 \mathrm{~m}$ can be finally calculated, as shown in Figs. 19 and 20. The results show that when the proportion of micropores is large, the absolute adsorption amount is also large, and it has a big contribution to the total gas amount. However, under other conditions, the free amount dominates the total gas amount. Based on this figure, we can quickly determine the absolute adsorption amount, the free gas amount, and the total gas amount of OM when we know the composition of organic pores in the OM. Thus, these charts can help us to evaluate the quality of a reservoir.

\section{Summary}

In this paper, six types of organic pores were studied based on the CCSIM-TSS method and the digital core compressibility method. After analysis of the numerical results, some important conclusions were obtained as follows:

1) The organic pores in the Longmaxi Formation marine shales can be classified into six types based on the morphology of nanoscale pores. 
The pore-size distribution and connectivity of the typical organic pores change greatly under deep reservoir conditions. As the depth of the reservoir increases, the size and connectivity of organic pores decrease.

2) Investigation of the gas transport propertie of OM with different proportion of micropores, mesopores, and macropores demonstrates that the permeability of OM has positive correlations with the biquadratic of the proportion of macropores and the square of the proportion of mesopores. However, the value of kapp/kd has a negative correlation with the square of the proportion of macropores and has a linear relationship with the proportion of mesopores.

3) The regularities of the absolute adsorption amount and the free gas amount of the typical organic pores were investigated. The absolute adsorption amount of OM decreases with the proportion of macropores, whereas the free gas amount of OM increases with the proportion of macropores. The chart of the total gas amount of OM with different compositions of organic pores are given at the depth of $1000 \mathrm{~m}$ and $4000 \mathrm{~m}$, which can help us to estimate the gas-bearing capacity of $\mathrm{OM}$ and preliminarily understand the quality of shale reservoirs.

In sum the structure, the apparent permeability and the gas-bearing property of OM with typical organic pores are investigated carefully and thoroughly. It should pointed out that the focus of our paper is on organic pores, and in the near future the gas-bearing property in inorganic pores will be studied and reported.

\section{Acknowledgements}

This work is supported by the National Natural Science Foundation of China (Grant No. 41690132 and 41872163), the Strategic Priority Research Program of the Chinese Academy of Sciences (Grant No. XDA14010304), and the Major National Science and Technology Special Program of China (Grant No. 2017ZX05037-001).

\section{Appendix A. Supplementary data}

Supplementary data related to this article can be found at https:// doi.org/10.1016/j.marpetgeo.2019.08.030.

\section{References}

Akkutlu, I.Y., Efendiev, Y., Savatorova, V., 2015. Multi-scale asymptotic analysis of gas transport in shale matrix. Transp. Porous Media 107, 235-260.

Akkutlu, I.Y., Fathi, E., 2012. Multiscale gas transport in shales with local kerogen heterogeneities. SPE J. 17, 1002-1011.

Chen, C., 2016. Multiscale imaging, modeling, and principal component analysis of gas transport in shale reservoirs. Fuel 182, 761-770.

Chen, M., Kang, Y., Li, X., Wang, W., Yang, B., Liu, H., 2016. Investigation of multi-scale gas transport behavior in organic-rich shale. J. Nat. Gas Sci. Eng. 36, 1188-1198.
Curtis, J.B., 2002. Fractured shale-gas systems. AAPG Bull. 86, 1921-1938.

Darabi, H., Ettehad, A., Javadpour, F., Sepehrnoori, K., 2012. Gas flow in ultra-tight shale strata. J. Fluid Mech. 7 (10), 641-658.

Dong, J.J., Hsu, J.Y., Wu, W.J., Shimamoto, T.J., Hung, H.E., Yeh, C.Y., Wu, H., Sone, H., 2010. Stress dependence of the permeability and porosity of sandstone and shale from TCDP Hole-A. Int. J. Rock Mech. Min. Sci. 47, 1141-1157.

Fathi, E., Akkutlu, I.Y., 2011. Lattice Boltzmann method for simulation of shale gas transport in kerogen. SPE J. 18, 27-37.

Guo, C.H., Xu, J.C., Wu, K.L., Wei, M.Z., Liu, S.Y., 2015. Study on gas flow through nano pores of shale gas reservoirs. Fuel 143, 107-117.

Javadpour, F., Fisher, D., Unsworth, M., 2007. Nanoscale gas flow in shale gas sediments. J. Can. Pet. Technol. 46, 55-61.

Javadpour, F., 2009. Nanopores and apparent permeability of gas flow in mud rocks (shales and siltstone). J. Can. Pet. Technol. 48, 16-21.

Jiang, W.B., Lin, M., et al., 2017. Parameter determination using 3D FIB-SEM images for development of effective model of shale gas flow in nanoscale pore cluster. Transp. Porous Media 1, 5-25.

Jiang, W.B., Lin, M., 2018. Molecular dynamics investigation of conversion methods for excess adsorption amount of shale gas. J. Nat. Gas Sci. Eng. 49, 241-249.

Ji, L.L., Lin, M., Jiang, W.B., et al., 2018. An improved method for reconstructing the digital core model of heterogeneous porous media. Transp. Porous Media 121 (2), 389-406.

Ji, L.L., Lin, M., Jiang, W.B., Cao, G.H., 2019. A hybrid method for reconstruction of three-Dimensional heterogeneous porous media from two-dimensional images. J. Asian Earth Sci. https://doi.org/10.1016/j.jseaes.2018.04.026.

Liu, H.L., Guo, W., Fang, C.H., Wang, H.Y., 2015. A study on geological characteristics of marine shale gas reservoir in Southern China. Energy Explor. Exploit. 33 (3), 299-308.

Loucks, R.G., Reed, R.M., Ruppel, S.C., Jarvie, D.M., 2009. Morphology, genesis, and distribution of nanometer-scale pores in siliceous mudstones of the Mississippian Barnett shale. J. Sediment. Res. 79, 848-861.

Lu, X.C., Li, F.C., Watson, A.T., 1995. Adsorption measurements in Devonian shales. Fuel $74,599-603$.

Naraghi, M.E., Javadpour, F., 2015. A stochastic permeability model for the shale-gas systems. Int. J. Coal Geol. 140, 111-124.

Reed, R.M., Ruppel, S.C., 2012. Pore morphology and distribution in the cretaceous eagle ford shale, south Texas, USA. Gulf Coast Assoc. Geol. Soc. Trans. 62, 599-604.

Ross, D.J., Bustin, R.M., 2009. The importance of shale composition and pore structure upon gas storage potential of shale gas reservoirs. Mar. Pet. Geol. 26, 916-927.

Rouquerol, J., Avnir, D., Fairbridge, C.W., Unger, K.K., 1994. Recommendations for the characterization of porous solids (technical report). Pure Appl. Chem. 66 (8), 1739-1758.

Sheng, M., Li, G., Tian, S., Huang, Z., Chen, L., 2016. A fractal permeability model for shale matrix with multi-scale porous structure. Fractals 24, 1650002.

Sheng, G.L., Javadpour, F.Z., Su, Y.L., 2018. Effect of microscale compressibility on apparent porosity and permeability in shale gas reservoirs. Int. J. Heat Mass Transf. $120,56-65$.

Song, W., Yao, J., Li, Y., Sun, H., Zhang, L., Yang, Y., et al., 2016. Apparent gas permeability in an organic-rich shale reservoir. Fuel 181, 973-984.

Wang, J., Chen, L., Kang, Q., Rahman, S.S., 2016. Permeability prediction of organic shale with generalized lattice Boltzmann model considering surface diffusion effect. Fuel 181, 478-490.

Wasaki, A., Akkutlu, I.Y., 2015. Permeability of organic-rich shale. SPE J. 20, 1384-1396.

Wu, K.L., Chen, Z.X., Li, X.F., Guo, C.H., Wei, M.Z., 2016. A model for multiple transport mechanisms through nanopores of shale gas reservoirs with real gas effect-adsorption-mechanic coupling. Int. J. Heat Mass Transf. 93, 408-426.

Xiong, F., Jiang, Z., Li, P., Wang, X., Bi, H., Li, Y., et al., 2017. Pore structure of transitional shales in the Ordos Basin, NW China: effects of composition on gas storage capacity. Fuel 206, 504-515.

Yang, F., Ning, Z.F., Liu, H.Q., 2014. Fractal characteristics of shales from a shale gas reservoir in the Sichuan basin, China. Fuel 115, 378-384.

Yi, Z.X., Lin, M., Jiang, W.B., et al., 2017. Pore network extraction from pore space images of various porous media systems. Water Resour. Res. 53 (4), 3424-3445. 